\title{
LARGE-TIME BEHAVIOUR OF THE ENTROPY SOLUTION OF A SCALAR CONSERVATION LAW WITH BOUNDARY CONDITIONS
}

\author{
BY
}

S. MARTIN (INSA de Lyon Institut Camille Jordan, CNRS UMR 5208, Bât. L. de Vinci, 21 av. Jean Capelle, F-69621 Villeurbanne cedex, France)

AND

J. VOVELLE (ENS Cachan Antenne de Bretagne IRMAR, CNRS UMR 6625, Avenue Robert Schuman, Campus de Ker Lann, F-35170 Bruz, France)

\begin{abstract}
We study the large-time behaviour of the entropy solution of a scalar conservation law with boundary conditions. Under structural hypotheses on the flux of the equation, we describe the stationary solutions and show the convergence of the entropy solution to a stationary one. Numerical tests illustrate the theoretical results.
\end{abstract}

\section{Introduction.}

1.1. Statement of the problem. We study the large-time behaviour of the solution of the one-dimensional non-autonomous conservation law with boundary conditions

$$
\begin{cases}u_{t}(x, t)+(A(x, u))_{x}(x, t)=0, & (x, t) \in(0,1) \times(0,+\infty), \\ u(x, 0)=u_{0}(x), & x \in(0,1), \\ u(0, t)=\bar{u}_{0}(t), & t>0, \\ u(1, t)=\bar{u}_{1}(t), & t>0 .\end{cases}
$$

This study was initially motivated by the analysis of a problem which arises in lubrication theory. The behaviour of a thin film flow of two non-miscible fluids between two surfaces in relative motion has been derived by Paoli [Pao03] and further developped by Bayada, Martin and Vázquez [BMV05]. In these papers, it is stated that the saturation of the reference fluid satisfies a scalar conservation law as the one described in problem (1) (the so-called generalized Buckley-Leverett equation). As was already pointed out in [BMV05], this model is of great interest in lubrication theory, especially for the understanding of cavitation phenomena in devices such as journal bearings of infinite width. Cavitation, which is defined as the rupture of the continuous liquid lubricant film due to the formation of gaseous bubbles (see [Do63, EA75] for precise physical explanations)

Received February 1, 2006.

2000 Mathematics Subject Classification. Primary 35L65, 35B40.

E-mail address: sebastien.martin@insa-lyon.fr

E-mail address: julien.vovelle@bretagne.ens-cachan.fr 
may be approached using the generalized Buckley-Leverett model, by considering the bifluid as a liquid-gas mixture. Finally, for practical convenience, it is also relevant to focus on steady-state regimes which are particularly studied in the field of tribology.

References on the large-time behaviour of the entropy solution of scalar conservation laws with boundary conditions are quite few, to our knowledge. See the work of Mascia and Terracina [MT99] and references therein in case the equation has a source term. On the contrary, numerous works are devoted to the study of the large-time behaviour of the entropy solution of a scalar conservation law posed on the whole space (Cauchy problem). See in particular the results of decay of the entropy solution to $N$-waves, as studied in [Lax57, IO60, DiP75, LP84, Daf85, Kim03]. Consider also the studies on the stability of profiles for scalar conservation laws on the line, in particular in [KM85, MN94, LN97, FS01, Ser04]. We emphasize in particular the works of Serre and coworkers, who developped a general strategy for the study of the stability of profiles of scalar conservation laws which inspired us for the present analysis.

To the specific framework of lubrication theory is associated precise hypotheses on the flux $A$ and on the data. Among these hypotheses, we have selected the following ones:

Assumption 1 (Data). The boundary and initial data satisfy the following:

(i) initial conditions:

(ii) boundary conditions:

$$
u_{0} \in L^{\infty}(0,1), \quad 0 \leq u_{0} \leq 1 \text { a.e. }
$$

$$
\bar{u}_{0}=\bar{u}_{1}=\bar{u} \in[0,1] .
$$

REMARK 1. The unknown $u$, as a saturation, takes value in $[0,1]$, and so do the data. Furthermore $\bar{u}_{0}$ and $\bar{u}_{1}$ are constant functions: this allows us to deal with realistic boundary conditions in infinite journal bearings as will be discussed further.

Assumption 2 (Flux function). The flux function $A \in \mathcal{C}^{1}\left([0,1]^{2}\right)$ satisfies the following properties:

$$
\begin{aligned}
& \forall x \in[0,1], A(x, \cdot)=A(1-x, \cdot), A(x, 0)=0, A(x, 1)=Q \geq 0, \\
& \forall x \in[0,1], \exists \alpha(x) \in(0,1],\left\{\begin{array}{l}
A_{u}(x, \cdot)>0 \text { on }[0, \alpha(x)), \\
A_{u}(x, \cdot)<0 \text { on }(\alpha(x), 1],
\end{array}\right. \\
& \forall u \in[0,1], x \mapsto A(x, u) \text { is non-increasing on }[0,1 / 2] .
\end{aligned}
$$

REMARK 2 (Lubrication theory). In lubrication, a plain cylindrical journal bearing is made of an inner rotating cylinder and an outer cylinder. The two cylinders are closely spaced and the annular gap between the two cylinders is filled with some lubricant. The radial clearance is very small, typically $\Delta r / r=10^{-3}$ for oil lubricated bearings so that the smallness of this ratio allows for a Cartesian coordinate to be located on the bearing surface. When cavitation occurs, the two-phase flow is described by problem (1), with a flux $A(x, u)=Q f(u)+v_{0} H(x) u(1-f(u)$ ), where $u$ denotes the (unknown) saturation of the liquid phase, $f$ is an $\mathcal{S}$-shaped function (with $f(0)=0, f(1)=1$ ), $H$ is the normalized converging-diverging gap between the two close surfaces in relative motion, $v_{0}$ is the shear velocity of the lower surface (the upper one being fixed) and $Q$ is the flow input. Hypothesis (3) (see Assumption 1) is natural in infinite journal bearings: indeed, the annular gap is filled with some lubricant at the supply groove which is 
located at the maximum gap [BMV05], the saturation of the liquid phase being imposed in this located area (corresponding to each extremity in the Cartesian coordinates). Let us emphasize that the non-autonomous property of the flux function comes from the shear effects and/or the converging-diverging profile of the normalized gap. This affects the mathematical analysis of the large-time behaviour of the solution (mainly because balance effects appear, owing to the dependence on $x$ of the flux and the possibility of more stationary profiles than in the case of an autonomous flux). However the non-zero shear velocity of the lower surface and the specific profile of the gap are characteristic features of realistic lubrication regimes.

REMARK 3 (Model case). A model case of a flux function is given by

$$
A(x, u)=Q u+H(x) u(1-u)
$$

where, according to the strength of $H(x) / Q, \alpha(x)=1(A(x, \cdot)$ increasing on $[0,1])$ or $\alpha(x) \in(0,1)(A(x, \cdot)$ has one strict maximum at $\alpha(x))$.

Remark 4. Hypothesis (3) can be relaxed ((2) still being satisfied). Notice also that, under hypotheses (2) and (4)-(6), problem (1) models two-phase flows in porous media under a gravity field (see, e.g., [Kaa99, EGV03]) or two-phase flow in a pipe [EGV03].

To achieve the discussion on hypotheses (2)-(6), let us emphasize the necessity to restrict the possible shapes of the data and of the flux. Indeed, consider the case where $A=0$. The solution of $(1)$ is then $u(x, t)=u_{0}(x)$, whose behaviour in large-time cannot be expected. Similarly, in the case where $A(x, u)=u$, the solution of (1) is, for $t>1$, $u(x, t)=\bar{u}_{0}(t-x)$ and $u(\cdot, t)$ converges to a function of $L^{\infty}(0,1 ;[0,1])$ in the $L^{1}$-norm at $t \rightarrow+\infty$ only if the means of $\bar{u}_{0}$ converge to a constant.

1.2. Structure of the paper and main results. The paper is organized as follows: in Section 2, we recall the notion of entropy solution (also sub- and super-solution) for problem (1) and its properties. In Section 3, we analyse the stationary solutions of problem (1) to give their description in Theorem 3. In Section 4, we study the large-time behaviour of the entropy solution of (1). Our main result, Theorem 4, states that the entropy solution to (1) converges to a stationary solution in $L^{1}$. In Section 5 , we present some numerical tests which illustrate the theoretical results.

\section{Entropy Solutions.}

2.1. Definitions. Let us start with some standard definitions and tools related to scalar conservation laws on bounded domains:

Definition 1. Assume that (2)-(4) hold. A function $u \in L^{\infty}((0,1) \times(0,+\infty))$ is said to be an entropy sub-solution (respectively super-solution) of problem (1) if for all $\kappa \in[0,1]$, for all $\varphi \in \mathcal{C}_{c}^{\infty}([0,1] \times[0,+\infty)), \varphi \geq 0$,

$$
\begin{array}{r}
\int_{0}^{1} \int_{0}^{\infty}(u-\kappa)^{ \pm} \varphi_{t}+\Phi^{ \pm}(x, u, \kappa) \varphi_{x}-\operatorname{sgn}_{ \pm}(u-\kappa) A_{x}(x, \kappa) \varphi d x d t \\
+\int_{0}^{1}\left(u_{0}-\kappa\right)^{ \pm} \varphi(0, x) d x \\
+\mathcal{L} \int_{0}^{\infty}\left(\left(\bar{u}_{0}-\kappa\right)^{ \pm} \varphi(t, 0)+\left(\bar{u}_{1}-\kappa\right)^{ \pm} \varphi(t, 1)\right) d t \geq 0
\end{array}
$$


The functions $u \mapsto(u-\kappa)^{ \pm}$are the so-called "semi-Kruzkov entropies" (see [Car99, Ser96, Vov02]), defined by

$$
(u-\kappa)^{+}=\left\{\begin{aligned}
u-\kappa, & \text { if } u \geq \kappa, \\
0, & \text { otherwise, }
\end{aligned} \quad \text { and } \quad(u-\kappa)^{-}=(\kappa-u)^{+} .\right.
$$

The functions $\Phi^{ \pm}(x, u, \kappa)$ are the corresponding "semi-Kruzkov fluxes" defined by

$$
\Phi^{ \pm}(x, u, \kappa)=\operatorname{sgn}_{ \pm}(u-\kappa)(A(x, u)-A(x, \kappa)),
$$

and $\mathcal{L}$ is a Lipschitz constant of $A(0, \cdot)$.

Definition 2. Assume that (2)-(4) hold. A function $u \in L^{\infty}((0,1) \times(0,+\infty))$ is said to be an entropy solution of problem (1) if it is a sub-solution and a super-solution of problem (1).

2.2. Properties of the entropy solution.

Theorem 1 (Existence, $L^{\infty}$ bound). Assume that (2)-(4) hold. The problem (1) admits at least one entropy solution $u \in L^{\infty}((0,1) \times(0,+\infty))$. Moreover, any entropy solution satisfies $0 \leq u \leq 1$ a.e.

Proof. The existence of entropy solutions of problem (1) is given by the convergence of approximations (parabolic or numeric ones); see the references [BLN79, Ott93, Ott96, MNRR96, Vov02, Mar05]. The bounds are directly deduced from the weak entropy formulation, using appropriate choices of entropy/flux pairs and values of $\kappa$.

Theorem 2 (Uniqueness [BLN79, Ott93, Mar05]). Assume that (2) and (4) hold. Let $u, v \in L^{\infty}((0,1) \times(0,+\infty))$ be, respectively, entropy sub- and super-solutions of problem (1) associated to respective data $u_{0}, \bar{u}_{0}, \bar{u}_{1}$ and $v_{0}, \bar{v}_{0}, \bar{v}_{1}$. Then, for all $\varphi \in \mathcal{C}_{c}^{\infty}([0,1] \times[0,+\infty)), \varphi \geq 0, u$ and $v$ satisfy the comparison inequality

$$
\begin{gathered}
\int_{0}^{1} \int_{0}^{\infty}(u-v)^{+} \varphi_{t}+\Phi^{+}(x, u, v) \varphi_{x} d x d t+\int_{0}^{1}\left(u_{0}-v_{0}\right)^{+} \varphi(0, x) d x \\
\mathcal{L} \int_{0}^{\infty}\left(\left(\bar{u}_{0}-\bar{v}_{0}\right)^{+} \varphi(t, 0)+\left(\bar{u}_{1}-\bar{v}_{1}\right)^{+} \varphi(t, 1)\right) d t \geq 0 .
\end{gathered}
$$

In particular, entropy solutions of problem (1) are uniquely determined by the initial and boundary data.

REMARK 5 (Localisation of the comparison). If $u, v \in L^{\infty}$ are entropy solutions in the interior of $(0,1) \times(0,+\infty)$, in the sense that $(7)$ holds for non-negative test functions $\varphi \in \mathcal{C}_{c}^{\infty}\left(\omega \times\left(t_{1}, t_{2}\right)\right)$ where $\omega$ is an open subset of $(0,1)$ and $0<t_{1}<t_{2}$, then (8) remains true on $\omega \times\left(t_{1}, t_{2}\right)$, i.e. (8) holds for non-negative $\varphi \in \mathcal{C}_{c}^{\infty}\left(\omega \times\left(t_{1}, t_{2}\right)\right)$.

We also have the following properties:

Lemma 1 (Continuity w.r.t. time). Assume that (2)-(4) hold. The entropy solution $u \in L^{\infty}((0,1) \times(0,+\infty))$ of problem $(1)$ is continuous from $[0,+\infty)$ in $L^{1}$ :

$$
u \in \mathcal{C}\left([0,+\infty) ; L^{1}(0,1)\right) \text { and } \lim _{t \rightarrow 0} u(t)=u_{0} \text { in } L^{1}(0,1) .
$$


ReMARK-Definition 1. In particular, and by Lemma 1, problem (1) defines a semigroup $S(t):\left(u_{0}, \bar{u}\right) \mapsto u(t)$ from $L^{\infty}(0,1 ;[0,1]) \times[0,1]$ into $L^{\infty}(0,1 ;[0,1])$, which associates to $\left(u_{0}, \bar{u}\right)$ the value at time $t$ of the entropy solution of problem (1) with initial datum $u_{0}$ and boundary data $\bar{u}_{0}=\bar{u}_{1}=\bar{u}$. We have

$$
\lim _{t \rightarrow 0} S(t)\left(u_{0}, \bar{u}\right)=u_{0} \text { in } L^{1}(0,1) .
$$

A classical consequence of $(8)$ is the fact that, for $\bar{u} \in[0,1], S(t)(\cdot, \bar{u})$ is non-expansive on $L^{1}$ : for every $u_{0}, v_{0} \in L^{\infty}(0,1 ;[0,1])$, one has

$$
\forall t>0, \quad\left\|S(t)\left(u_{0}, \bar{u}\right)-S(t)\left(v_{0}, \bar{u}\right)\right\|_{L^{1}(0,1)} \leq\left\|u_{0}-v_{0}\right\|_{L^{1}(0,1)} .
$$

In particular, $S(t)(\cdot, \bar{u})$ is continuous on $L^{\infty}(0,1 ;[0,1])$ endowed with the topology of the $L^{1}$-norm.

There are several ways to prove Lemma 1 . One way is by proving the convergence of an approximation in $\mathcal{C}\left([0, T] ; L^{1}(0,1)\right), T$ arbitrary positive time (this uses the uniqueness of the entropy solution): we refer to [Mar05] for such a result. The other way is by a direct proof on the basis of the entropy formulation. The critical step is then to prove the continuity at $t=0$ and to recover the initial condition; see [CR00, Vas01] for analysis of the problem of the initial layer in scalar conservation laws.

To complete Remark-Definition 1, we give the following proposition:

Proposition 1. Assume that (4) holds ((2) and (3) may be relaxed, the data being $L^{\infty}$ functions). Let $v \in L^{\infty}(0,1 ;[0,1]) \bigcap \mathcal{C}\left([0,+\infty) ; L^{1}(0,1)\right)$ (respectively $w \in$ $\left.L^{\infty}(0,1 ;[0,1]) \bigcap \mathcal{C}\left([0,+\infty) ; L^{1}(0,1)\right)\right)$ be an entropy sub- (resp. super-) solution of (1) with data $\left(v_{0}, \bar{v}\right)\left(\operatorname{resp} .\left(w_{0}, \bar{w}\right)\right)$ and let

$$
\left(u_{0}, \bar{u}\right) \in L^{\infty}(0,1 ;[0,1]) \times[\bar{v}, \bar{w}] .
$$

Let $S(t)$ be the semi-group defined in Remark-Definition 1. Then

$$
t \mapsto\left\|\left(v(t)-S(t)\left(u_{0}, \bar{u}\right)\right)^{+}\right\|_{L^{1}(0,1)} \text { and } t \mapsto\left\|\left(S(t)\left(u_{0}, \bar{u}\right)-w(t)\right)^{+}\right\|_{L^{1}(0,1)}
$$

are non-increasing. In particular, if $v_{0} \leq u_{0}$ (resp. $\left.u_{0} \leq w_{0}\right)$, then $v(t) \leq S(t)\left(u_{0}, \bar{u}\right)$ (resp. $\left.S(t)\left(u_{0}, \bar{u}\right) \leq w(t)\right)$ a.e. on $(0,1)$.

Proof. Proposition 1 is a direct consequence of Theorem 2: choose a test function $\varphi$ independent of $x$ to get, in the weak sense,

$$
\rho^{\prime}(t) \leq 0, \quad \rho(t)=\int_{0}^{1}\left(v(t)-S(t)\left(u_{0}, \bar{u}\right)\right)^{+} d x \leq 0 .
$$

Besides, $\rho$ is a continuous function; it is therefore non-increasing.

Lemma 2 (BV bound [BLN79]). Assume that (4) holds. Then there exists a non-negative function $C_{B V}$ from $\mathbb{R}_{+}$to $\mathbb{R}_{+}$such that, for $\bar{u} \in[0,1]$,

$$
\left\|S(t)\left(u_{0}, \bar{u}\right)\right\|_{B V(0,1)} \leq C_{B V}\left(\left\|u_{0}\right\|_{B V(0,1)}\right)
$$

for all $u_{0} \in B V(0,1) \cap L^{\infty}(0,1 ;[0,1])$.

In the context of Lemma $2, u(t):=S(t)\left(u_{0}, \bar{u}\right) \in B V(0,1)$ for every $t$. In particular the traces $u(0, t)$ and $u(1, t)$ make sense and the boundary conditions are given by the so-called BLN inequalities [BLN79]. 
Lemma 3 (Boundary conditions). Assume that (2)-(4) hold. Suppose $u_{0} \in B V(0,1) \cap$ $L^{\infty}(0,1 ;[0,1])$ and $\bar{u} \in[0,1]$. Then, a function

$$
u \in \mathcal{C}\left([0,+\infty) ; L^{1}(0,1)\right) \cap L^{\infty}(0,+\infty, B V(0,1))
$$

is the entropy solution of (1) (i.e. $\left.u(t)=S(t)\left(u_{0}, \bar{u}\right)\right)$ iff it satisfies the entropy inequalities inside $(0,1)$ : for all $\kappa \in[0,1]$, for all $\varphi \in \mathcal{C}_{c}^{\infty}((0,1) \times[0,+\infty)), \varphi \geq 0$,

$$
\begin{aligned}
\int_{0}^{1} \int_{0}^{\infty}(u-\kappa)^{ \pm} \varphi_{t}+\Phi^{ \pm}(x, u, \kappa) \varphi_{x} & -\operatorname{sgn}_{ \pm}(u-\kappa) A_{x}(x, \kappa) \varphi d x d t \\
& +\int_{0}^{1}\left(u_{0}-\kappa\right)^{ \pm} \varphi(0, x) d x \geq 0,
\end{aligned}
$$

and if, furthermore, it satisfies the BLN boundary conditions $([a, b]$ denotes the interval of extremities $a$ and $b$ ):

$$
\left\{\begin{array}{l}
\forall t>0, \forall \kappa \in\left[u(0, t), \bar{u}_{0}\right], \operatorname{sgn}(u(0, t)-\bar{u})(A(0, u(0, t))-A(0, \kappa)) \leq 0, \\
\forall t>0, \forall \kappa \in\left[u(1, t), \bar{u}_{1}\right], \operatorname{sgn}(u(1, t)-\bar{u})(A(1, u(1, t))-A(1, \kappa)) \geq 0 .
\end{array}\right.
$$

Let us pause here to analyse the above BLN condition. Denote temporarily $F(u)=$ $A(0, u)$. In case $F$ is monotonous on $[0,1]$, the BLN condition at $x=0$ translates the intuitive fact that for transport equations (e.g. with constant speed), the boundary condition is active (resp. inactive) on the part of the boundary that is enlightened (resp. in the dark); in other words, $u(0, t)=\bar{u}_{0}$ if $F$ is increasing while the whole range of values $[0,1]$ is admissible for $u(0, t)$ if $F$ is non-increasing. In the general case of regular function $F$, classical studies of boundary layers show that the admissibility of $u(0, t)$ with respect to the BLN condition at $x=0$ is equivalent to the solvability of the o.d.e.

$$
\begin{cases}\dot{w} & =F(w)-F(u(0, t)) \\ w\left(y_{0}\right) & =\bar{u}_{0} \\ w(+\infty) & =u(0, t)\end{cases}
$$

with $y_{0}=-\infty$ if $F\left(\bar{u}_{0}\right)=F(u(0, t))$ (complete orbit), $y_{0}=0$ otherwise (positive orbit). On the basis of this result, or by direct inspection of the BLN condition, one can see that this means

(1) either $\bar{u}_{0}$ is in a non-increasing part, say $\left[u_{-}, u_{+}\right]$, of the graph of $F$, and, as in the case where $F$ is monotonous, admissible values of $u(0, t)$ consists of the interval $\left[u_{-}, u_{+}\right]$,

(2) or $\bar{u}_{0}$ is in an increasing part of the graph of $F\left(F^{\prime}\left(\bar{u}_{0}\right)>0\right)$, in which case $u(0, t)$ is admissible if and only if the segment joining $\left(\bar{u}_{0}, F\left(\bar{u}_{0}\right)\right)$ to $(u(0, t), F(u(0, t)))$ has non-positive slope and does not intersect the graph of $F$ except at its extremities.

Since $A(0, \cdot)=A(1, \cdot)$ is an increasing function on $(0, \alpha(0))$ and a decreasing function on $(\alpha(0), 1)$, i.e. $A(0, \cdot)$ has the shape of a dome, we can associate to each $z \in[0,1]$ its $A$ conjugate $z^{A}$ defined by symmetry with respect to the height of the dome: $\alpha(0)^{A}=\alpha(0)$, $A\left(0, z^{A}\right)=A(0, z), z^{A} \neq z$ if $A(0, z) \geq Q$ and $z \neq \alpha(0), z^{A}=1$ if $0 \leq z \leq \theta(0)$. Then, at $x=0$, the BLN condition is equivalent to

$$
\left\{\begin{array}{l}
\text { either } 0 \leq \bar{u} \leq \alpha(0) \text { and } u(0, t)=\bar{u} \text { or } \bar{u}^{A} \leq u(0, t) \leq 1, \\
\text { or } \alpha(0) \leq \bar{u} \leq 1 \text { and } \alpha(0) \leq u(0, t) \leq 1
\end{array}\right.
$$


and, at $x=1$, the BLN condition is equivalent to

$$
\left\{\begin{array}{l}
\text { either } 0 \leq \bar{u} \leq \alpha(0) \text { and } 0 \leq u(1, t) \leq \alpha(0), \\
\text { or } \alpha(0) \leq \bar{u} \leq 1 \text { and } u(1, t)=\bar{u} \text { or } 0 \leq u(1, t) \leq \bar{u}^{A} .
\end{array}\right.
$$

\section{Stationary solutions.}

3.1. Constant flux. Recall that $S(t)$ is defined in Remark-Definition 1.

Definition 3. Assume that (2) and (4) hold. A function $w \in L^{\infty}(0,1 ;[0,1])$ is said to be a stationary solution of problem (1) if there exists a boundary datum $\bar{u} \in[0,1]$ such that $w=S(t)(w, \bar{u})$ for all $t>0$.

We begin the analysis of stationary solutions of (1) by the inversion of the equation $A(x, w)=c \in \mathbb{R}$.

Proposition 2. Let $w \in L^{\infty}(0,1 ;[0,1])$ be a stationary entropy solution of problem (1). Then $A(x, w)$ is constant a.e.

Proof. An entropy solution is a weak solution: $(A(x, w))_{x}=0$ in $\mathcal{D}^{\prime}((0,1) \times(0,+\infty))$. Since $w=w(x)$, this remains true in $\mathcal{D}^{\prime}(0,1)$. This last fact implies $A(x, w)=c \in \mathbb{R}$ by the following Lemma 4 (classical in the theory of distributions).

Lemma 4. Let $T \in \mathcal{D}^{\prime}(0,1)$ be such that $T^{\prime}=0$. Then $T=c \in \mathbb{R}$ (i.e. $T$ is a function of $L^{1}(0,1)$ which is constant a.e.).

We will also use the following lemma of the theory of distribution:

Lemma 5. Let $T \in \mathcal{D}^{\prime}(0,1)$ be such that $T^{\prime} \geq 0$. Then $T \in L_{\text {loc }}^{1}(0,1)$ and $T$ is a monotone non-decreasing function. If, besides, $T$ is a function with values in $\{0,1\}$, then there exists $x_{0} \in[0,1]$ such that $T(x)=\operatorname{sgn}_{+}\left(x-x_{0}\right)$.

Proof. The non-negative distribution $T^{\prime}$ is of order 0: for every compact subset $K$ of $(0,1)$, there exists a constant $C_{K}$ such that for every $\varphi \in \mathcal{D}(0,1)$ with $\operatorname{supp}(\varphi) \subset K$, $\left|T^{\prime}(\varphi)\right| \leq C_{K}|| \varphi \|_{L^{\infty}}$ (choose a non-negative $\psi \in \mathcal{D}(0,1)$ such that $\psi=1$ on $K$ and write that $T^{\prime}\left(\varphi \pm\|\varphi\|_{L^{\infty}} \psi\right)$ is non-negative (respectively non-positive) to get the result with $\left.C_{K}=T^{\prime}(\psi)\right)$. By the Riesz representation theorem $T^{\prime}$ is represented by a Radon measure $\mu$ on $(0,1)$. Let $x_{0} \in(0,1)$. By the Green formula for $B V$ functions, $T$ and the $B V$ function $\widetilde{T}: x \mapsto \mu\left(\left(x_{0}, 0, x\right)\right)$ have the same derivative in $\mathcal{D}^{\prime}(0,1)$. By Lemma 4, we conclude that $T=\widetilde{T}+C$, where $C$ denotes any constant. Since $B V(0,1) \subset L^{\infty}(0,1) \subset$ $L_{\text {loc }}^{1}(0,1), T$ is a function which is non-decreasing. If, furthermore, $T$ takes values in $\{0,1\}$, then, by monotony of $T, T^{-1}(0)$ and $T^{-1}(1)$ are connected, hence subintervals of $[0,1]$ and $T(x)=\operatorname{sgn}_{+}\left(x-x_{0}\right)$ with $x_{0}:=\sup T^{-1}(0)=\inf T^{-1}(1)$.

Now we examine the inversion of the identity $A(x, w)=c \in \mathbb{R}$. Denote by $q$ the value of $A(x, w)$. Under hypotheses (4)-(5), the equation $A(x, w)=q$ may have zero, one or two branches of solutions according to the value of $q$ (by branch of solution, we mean a continuous function $w$ from $[0,1]$ into itself such that $A(x, w(x))=q$ for all $x)$. Actually, we are looking at the intersection between the surface $\mathcal{S}:=\{A(x, w)=z\}$ of $[0,1]^{2} \times \mathbb{R}_{+}$ and the horizontal plane $z=q$ and two possible shapes of the surface $\mathcal{S}$ are possible 
according to the value of the parameter

$$
q_{\lim }:=\min \{A(x, \alpha(x)), x \in[0,1]\}=A(1 / 2, \alpha(1 / 2)) .
$$

If $q_{\lim }>Q$ (see Figs. 1 and 3), then the surface $\mathcal{S}$ is saddle-shaped with a saddle point at $\left(x=1 / 2, w=\alpha(1 / 2), z=q_{\lim }\right)$; if $q_{\lim }=Q$ (see Figs. 2 and 4 ), then the surface $\mathcal{S}$ is slide-shaped (it resembles the top of a small children's slide or part of a monkey-saddle).

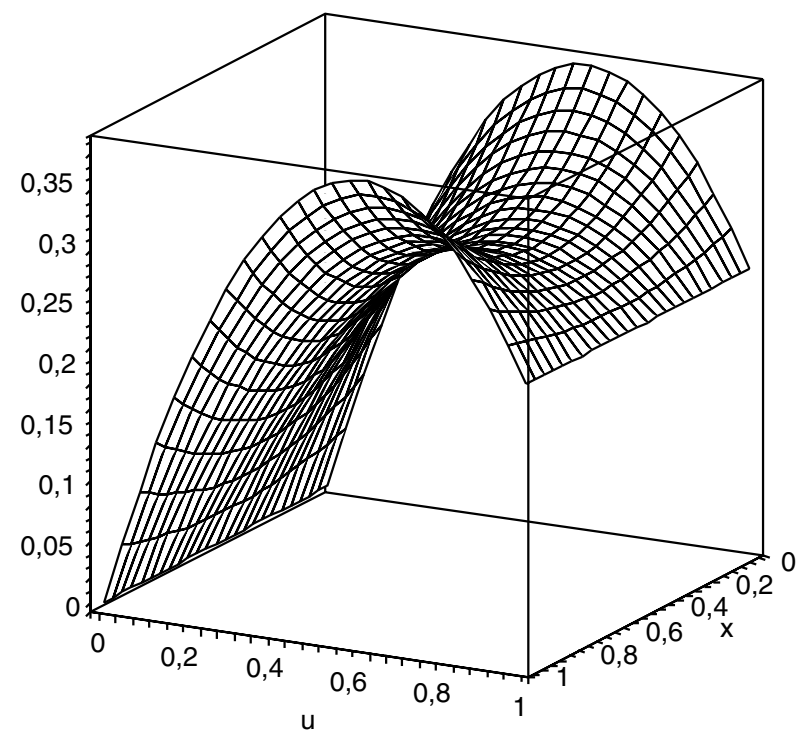

FIG. 1. Surface $\mathcal{S}$ (from behind) in the saddle case: $q_{\mathrm{lim}}>Q$.

Definition 4. If $q_{\text {lim }}>Q$, we say that we are in the saddle case; if $q_{\text {lim }}=Q$, we say that we are in the slide case.

The intersection of $\mathcal{S}$ and $\{z=q\}$ is described in the following lemma.

Lemma 6. Assume that (4)-(6) hold. We focus on the solutions of

$$
A(x, w(x))=q \in \mathbb{R}, \quad x \in[0,1], \quad w:[0,1] \rightarrow[0,1] \text { continuous },
$$

i.e. on the intersection $\mathcal{I}$ between the surface $\mathcal{S}:=\{A(x, w)=z\}$ and the horizontal plane $z=q$ in $[0,1]^{2} \times \mathbb{R}$. There are bifurcations at $q=0, Q, q_{\text {lim }}$ :

- if $q<0$, the intersection is empty;

- if $0 \leq q<Q$, the intersection $\mathcal{I}$ defines one continuous curve $w=\lambda(x)$;

- otherwise, we have the following subcases :

○ if $Q<q_{\text {lim }}$ (saddle case): for $Q \leq q \leq q_{\text {lim }}$, the intersection $\mathcal{I}$ defines two curves $w=\lambda(x)$ and $w=\mu(x)$ (with, by convention, $\lambda \leq \mu$ ); for 


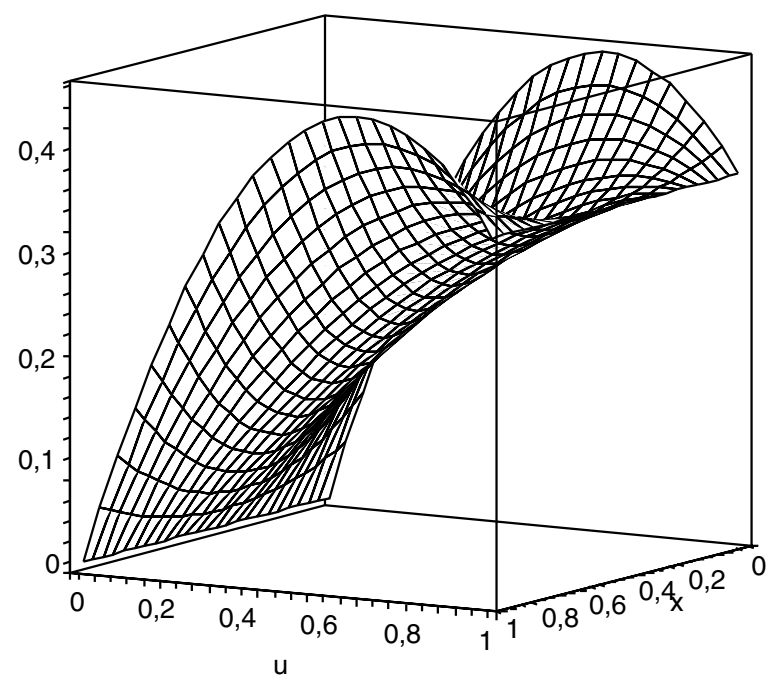

FIG. 2. Surface $\mathcal{S}$ (from behind) in the slide case $Q=q_{\text {lim }}$.

$q_{\lim }<q \leq A(0, \alpha(0))$, the intersection $\mathcal{I}$ consists of two cusps and for $q>A(0, \alpha(0))$ the intersection is empty;

○ if $Q=q_{\text {lim }}$ (slide case), then, for $Q=q=q_{\text {lim }}$, the intersection is parametrized by two curves $w=\lambda(x)$ and $w=\mu(x)$ which coincide on an interval centered on $x=1 / 2$. Actually, $\mu$ is the constant function 1. If $q>q_{\mathrm{lim}}$, there are no solutions to (14).

Notice that, in the saddle case, the curves $\lambda$ and $\mu$ are distinct if $Q \leq q<q_{\text {lim }}$ and intersect each other when $q=q_{\text {lim }}$. Therefore, whatever the case is (saddle or slide), the intersection $\mathcal{I}$ consists of two intersecting branches exactly when $q=q_{\text {lim }}$ (which will be the value of the flux for which the large-time behaviour of the entropy solution is the more complex one).

Notice also that, in the saddle case, when $q_{\text {lim }}<q \leq A(0, \alpha(0))$, the two cusps of $\mathcal{I}$ can be parametrized with the help of two functions $\lambda(x) \leq \mu(x)$ defined on $[0,1] \backslash\left(x_{q}, 1-x_{q}\right)$ for a given $x_{q} \in(0,1 / 2)$ : this will be used later.

To continue the analysis of the stationary solutions to (1), notice that, in case the equation $A(x, w(x))=q$ has more than one elementary solution, any function constructed by introducing jumps between two elementary solutions is also a solution, in $L^{\infty}((0,1) ;[0,1])$, of the equation $A(x, w(x))=q$. To specify the set of stationary solutions, we use the entropy condition and the boundary conditions. 
3.2. Entropy and boundary conditions. Lemma 6 shows that, for $0 \leq q \leq q_{\text {lim }}$, the equation $A(x, w(x))=q$ has one or two branches of solutions. We call them $\lambda$ and $\mu$, $\lambda \leq \mu$, with the convention $\lambda=\mu$ if there is actually only one branch.

Lemma 7. Assume that (4)-(6) hold. Let $q \in\left[0, q_{\text {lim }}\right]$. Let $\lambda$ and $\mu$ be the branches of solutions to (14). Let us introduce an increasing jump between $\lambda$ and $\mu$ by defining

$$
w_{z}:=\lambda \mathbf{1}_{[0, z)}+\mu \mathbf{1}_{(z, 1]}
$$

for $0 \leq z \leq 1$, and (in the case $\lambda$ and $\mu$ intersects each other) let us introduce a second increasing jump by defining

$$
w_{z, z^{\prime}}:=w_{z} \mathbf{1}_{[0,1 / 2]}+w_{z^{\prime}} \mathbf{1}_{[1 / 2,1]}
$$

for $0 \leq z \leq 1 / 2 \leq z^{\prime} \leq 1$. Then any stationary solution $w$ to problem (1) such that $A(x, w(x))=q$ a.e. is one of the $w_{z}, z \in[0,1]$, or one of the $w_{z, z^{\prime}}, 0 \leq z \leq 1 / 2 \leq z^{\prime} \leq 1$, the last possibility being restricted to the case where $\lambda$ and $\mu$ intersect each other (i.e. $\left.0 \leq q \leq q_{\lim }\right)$.

Proof. In the case where $\lambda=\mu$, the statement and its proof are obvious. We suppose therefore that $\lambda$ and $\mu$ are distinct functions.

- Let us first suppose that $Q \leq q<q_{\text {lim }}$. Let $w \in L^{\infty}(0,1 ;[0,1])$ be a stationary entropy solution of (1) with flux $q$. Choose $\tilde{q} \in\left(q, q_{\lim }\right)$. We denote by $\tilde{\lambda}, \tilde{\mu}$ the corresponding stationary solutions constructed as above. On the one hand, by comparison (see Remark 5 - we consider the case $\omega=(0,1)$ here), we have

$$
\int_{0}^{1} \Phi^{+}(x, \tilde{\lambda}, w) \varphi_{x} d x \geq 0
$$

for all non-negative $\varphi \in \mathcal{C}_{c}^{\infty}(0,1)$. It follows that $\partial_{x} \Phi^{+}(x, \tilde{\lambda}, w) \leq 0$ in $\mathcal{D}^{\prime}(0,1)$. On the other hand, we have

$$
\Phi^{+}(x, \tilde{\lambda}, w)=\operatorname{sgn}_{+}(\tilde{\lambda}-w)(A(x, \tilde{\lambda})-A(x, w))=\operatorname{sgn}_{+}(\tilde{\lambda}-w)(\tilde{q}-q)
$$

and, therefore, $\partial_{x} \operatorname{sgn}_{+}(\tilde{\lambda}-w) \leq 0$ in $\mathcal{D}^{\prime}(0,1)$. Now, by Lemma 5 , there exists some $x_{0} \in[0,1]$ such that

$$
\operatorname{sgn}_{+}(\tilde{\lambda}-w)(x)=1-\operatorname{sgn}_{+}\left(x-x_{0}\right), \quad x \in(0,1) .
$$

For every $x \in[0,1], u \mapsto \operatorname{sgn}_{+}(\tilde{\lambda}(x)-u) A(x, u)$ is a homeomorphism from $[0, \tilde{\lambda}(x))$ onto $[0, \tilde{q})$; therefore the identity

$$
\operatorname{sgn}_{+}(\tilde{\lambda}(x)-w(x)) A(x, w(x))=q, \quad x \in\left(0, x_{0}\right),
$$

implies that $w=\lambda$ on $\left(0, x_{0}\right)$. Similarly, we would prove that there exists some $x_{1} \in[0,1]$ such that $w=\mu$ on $\left(x_{1}, 1\right)$. Finally, since $\lambda<\mu$, we have $x_{0} \leq x_{1}$. If $x_{0}<x_{1}$, then $\tilde{\lambda} \leq w \leq \tilde{\mu}$ on $\left(x_{0}, x_{1}\right)$, which contradicts $A(x, w(x))=q$ a.e. Therefore $x_{0}=x_{1}=z \in[0,1]$ and $w=w_{z}$.

- Let us suppose that $q=q_{\mathrm{lim}}$. The proof of the result is similar, except that the solutions $\tilde{\lambda}$ and $\tilde{\mu}$ are not defined everywhere on $(0,1)$ : let $w \in L^{\infty}(0,1 ;[0,1])$ be a stationary entropy solution of (1) with flux $q$. Suppose that $z_{*}$ is the smallest element of $[0,1 / 2]$ such that $\lambda=\mu$ on $\left[z_{*}, 1-z_{*}\right]$. Since $\lambda$ and $\mu$ are 
distinct, we have $0<z_{*} \leq 1 / 2$. By minimality of $z_{*}$, we have, for any $\varepsilon>0$, $\lambda\left(z_{*}-\varepsilon\right)<\mu\left(z_{*}-\varepsilon\right)$. In particular,

$$
A\left(z_{*}-\varepsilon, \alpha\left(z_{*}-\varepsilon\right)\right)>q_{\text {lim }} .
$$

Now, let us choose $\tilde{q}$ such that

$$
q_{\lim }<\tilde{q}<A\left(z_{*}-\varepsilon, \alpha\left(z_{*}-\varepsilon\right)\right) .
$$

Then $\tilde{\lambda}$ exists on $\left[0, z_{*}-\varepsilon\right]$ and, as in the proof of the case $Q \leq q<q_{\text {lim }}$ (using Remark 5 with $\left.\omega=\left(0, z_{*}-\varepsilon\right)\right)$, we can show that $\operatorname{sgn}_{+}(\tilde{\lambda}-w)$ is non-increasing on $\left(0, z_{*}-\varepsilon\right)$. Now, for every $x \in\left[0, z_{*}-\varepsilon\right], u \mapsto \operatorname{sgn}_{+}(\tilde{\lambda}(x)-u) A(x, u)$ is a homeomorphism from $[0, \tilde{\lambda}(x))$ onto $[0, \tilde{q})$ so that there exists $x_{\varepsilon} \in\left[0, z_{*}\right]$ such that $w=\lambda$ on $\left(0, x_{\varepsilon}\right)$. Letting $\varepsilon$ tend to 0 , we infer that there exists $x_{0} \in\left[0, z_{*}\right]$ such that $w=\lambda$ on $\left(0, x_{0}\right)$. Similarly, we show that there exists $x_{1} \in\left[0, z_{*}\right]$ such that $w=\mu$ on $\left(x_{1}, z_{*}\right)$. If $x_{0}<x_{1}$, we have $x_{0}<x_{1}-\varepsilon$ for some $\varepsilon>0$. Then choosing again some $\tilde{q} \in\left(q_{\lim }, A\left(z_{*}-\varepsilon, \alpha\left(z_{*}-\varepsilon\right)\right)\right)$, we see that, necessarily, $\tilde{\lambda} \leq w \leq \tilde{\mu}$ on $\left(x_{0}, x_{1}\right)$, which contradicts $A(x, w(x))=q$ a.e. In conclusion, there exists $z \in\left[0, z_{*}\right]$ such that $w=w_{z}$ on $[0,1 / 2]$. Similarly, we can show that there exists $z^{\prime} \in\left[1-z_{*}, 1\right]$ such that $w=w_{z^{\prime}}$ on $[1 / 2,1]$. This shows that $w=w_{z, z^{\prime}}$ and concludes the proof of the lemma.

Every function $w_{z}$ or $w_{z, z^{\prime}}$ is an entropy solution inside $(0,1)$ and admits traces at $x=0$ and $x=1$. It is then straightforward to use the BLN condition (12)-(13) to characterize the stationary solutions to (1). To depict the stationary solutions with repect to a graph $((x, u(x))$, or $(x, u, z))$ we say that a piecewise continuous function $u:[0,1] \rightarrow \mathbb{R}$ starts (resp. ends) at $\lambda$ if $u(0+)=\lambda(0)$ (resp. $u(1-)=\lambda(0)$ ), similarly with respect to $\mu$.

Theorem 3. Assume that (2)-(6) hold. Let $u \in L^{\infty}((0,1) ;[0,1])$ be a stationary solution of (1) with boundary datum $\bar{u}$ (i.e. $S(t)(u, \bar{u})=u, \forall t>0$ ) and set $\bar{q}:=A(0, \bar{u})$. With the notation of Lemma 7 , we have the following results.

1. The flux $A(x, u(x))$ is equal a.e. to a constant $q$ and $u$ is a function $w_{z}$ or $w_{z, z^{\prime}}$.

2. According to the value of $\bar{q}$, we have

- if $\bar{q} \leq q_{\text {lim }}$, then $q=\bar{q}$, i.e. $\bar{u}=\lambda(0)$ or $\mu(0)$ : if $\bar{u}=\lambda(0)$, then $u$ ends at $\lambda(0)$; if $\bar{u}=\mu(0)$, then $u$ starts at $\mu(0)$;

- if $\bar{q}>q_{\mathrm{lim}}$, then $q=q_{\mathrm{lim}}$ and $u$ starts at $\mu(0)$ and ends at $\lambda(0): u=w_{0,1}=$ $\lambda \mathbf{1}_{[1 / 2,1]}+\mu \mathbf{1}_{[0,1 / 2]}$.

Observe that, in the case $\bar{q} \leq q_{\text {lim }}, u$ ends at $\lambda(0)$ if, and only if, we have $(u=\lambda$ and $\left.q=\bar{q}<q_{\text {lim }}\right)$ or $\left(u=\lambda \mathbf{1}_{[0, z) \cup[1 / 2,1]}+\mu \mathbf{1}_{(z, 1 / 2]}=w_{z, 1}\right.$ for $0 \leq z \leq 1 / 2$ and $\left.q=\bar{q}=q_{\text {lim }}\right)$ and $u$ starts at $\mu$ if, and only if, we have $\left(u=\mu\right.$ and $\left.q=\bar{q}<q_{\text {lim }}\right)$ or $\left(u=\lambda \mathbf{1}_{\left[1 / 2, z^{\prime}\right)}+\mu \mathbf{1}_{[0,1 / 2] \cup\left(z^{\prime}, 1\right]}=w_{0, z^{\prime}}\right.$ for $1 / 2 \leq z^{\prime} \leq 1$ and $\left.q=\bar{q}=q_{\text {lim }}\right)$. Notice also that discontinuous stationary solutions exist only in the case $\bar{q}=q_{\text {lim }}$.

3.3. Sub- and super-stationary solutions. We have described the stationary solutions to problem (1). In the analysis of the large-time behaviour of the entropy solution in the 
next section, we use the comparison principle and need to know enough sub- and superstationary solutions to problem (1) to analyse the $\omega$-limit sets of the trajectories. To that purpose, we proceed as in the preceding paragraphs: first we examine the solutions inside $(0,1)$ and focus on the entropy condition, and then we examine the boundary conditions.

Let us build some sets of sub- and super-stationary solutions to (1): given $\bar{u} \in[0,1]$, we set $\bar{q}:=A(0, \bar{u})$, we denote by $q:=\min \left(\bar{q}, q_{\text {lim }}\right)$ the flux of the corresponding stationary solutions to (1) (see Theorem 3) and we introduce the function

$$
\hat{\mu}:= \begin{cases}1, & \text { if } q<Q \\ \mu, & \text { if } q \geq Q\end{cases}
$$

(the functions $\lambda$ and $\hat{\mu}$ then describe the intersection between the plane $\{z=q\}$ and the surface $\hat{\mathcal{S}}:=\mathcal{S} \cup\{x=1\} \times\{u=1\} \times\{0 \leq z \leq Q\}$ which naturally completes the graph $\mathcal{S}$ of $A$ in the space $(x, u, z))$.

Piecewise continuous functions $u$ with increasing jumps and such that $x \mapsto A(x, u(x))$ is non-increasing (resp. non-decreasing) provide sub- (resp. super-) solutions of (1) inside $(0,1)$. Such functions admit traces at $x=0$ and $x=1$ and, as a consequence of Definition 1, are sub- (resp. super-) solutions of (1) as soon as they satisfy

$$
\begin{array}{r}
-\Phi^{ \pm}(0, u(0+), k)+\mathcal{L}(\bar{u}-k)^{ \pm} \geq 0, \quad 0 \leq k \leq 1, \\
\Phi^{ \pm}(1, u(1-), k)+\mathcal{L}(\bar{u}-k)^{ \pm} \geq 0, \quad 0 \leq k \leq 1 .
\end{array}
$$

Straightforward computations then show that, in case $0 \leq \bar{u} \leq \alpha(0)$, the sub-stationary solutions are subjected to start from the region $\{A(0, u(0+)) \leq \bar{q}\}$ and end in $[0, \alpha(0)]$ while, in the case $\alpha(0) \leq \bar{u}$, the sub-stationary solutions are subjected to end in $[0, \bar{u}]$.

Similarly, in case $0 \leq \bar{u} \leq \alpha(0)$, the super-stationary solutions are subjected to start from the region $[\bar{u}, 1]$ while, in the case $\alpha(0) \leq \bar{u}$, they are subjected to start from $[\alpha(0), 1]$ and end in $\{A(0, u(1-)) \leq \bar{q}\}$.

With these elements at hand, we can prove the following lemma:

Lemma 8. Assume that (4)-(6) hold. Set $\bar{q}:=A(0, \bar{u})$ and $q:=\min \left(\bar{q}, q_{\lim }\right)$. Let $\lambda$ and $\mu$ be the elementary solutions of $A(x, w(x))=q$ defined in Lemma 6 . Set $\hat{w}_{z}=$ $\lambda \mathbf{1}_{[0, z)}+\hat{\mu} \mathbf{1}_{(z, 1]}$ with

$$
\hat{\mu}:= \begin{cases}1, & \text { if } q<Q, \\ \mu, & \text { if } q \geq Q .\end{cases}
$$

- If $\bar{q}<q_{\lim }$, then the functions $\hat{w}_{z}, 0 \leq z \leq 1$, are sub-stationary solutions of problem (1) if $\bar{u}=\mu(0)$ and super-stationary solutions of problem (1) if $\bar{u}=\lambda(0)$.

- If $\bar{q}=q_{\lim }$, then the functions $w_{0, z^{\prime}}, 1 / 2 \leq z^{\prime} \leq 1$, are super-solutions while the functions $w_{z, 1}, 0 \leq z \leq 1 / 2$ are sub-solutions of problem (1).

Additionally, if $\bar{q}=q_{\mathrm{lim}}$, then the functions

$$
w_{1 / 2,1 / 2}=\lambda \mathbf{1}_{[0,1 / 2]}+\mu \mathbf{1}_{[1 / 2,1]}, \quad w_{0,1}=\lambda \mathbf{1}_{[1 / 2,1]}+\mu \mathbf{1}_{[0,1 / 2]}
$$

are, respectively, super- and sub-solution of problem (1). 
4. Large-time asymptotic behaviour. Fix $\bar{u} \in[0,1]$. Let $X_{0}$ denote the set $X_{0}:=$ $L^{\infty}(0,1 ;[0,1])$ endowed with the topology of the $L^{1}$-norm $\left(X_{0}\right.$ is a Banach space for it is closed in $L^{1}$ since any convergent sequence of $L^{1}$ has a subsequence which converges almost everywhere). Denote by $S_{\bar{u}}(t):=S(\cdot, \bar{u})$ the semi-group which, to any $u_{0} \in X_{0}$, associates the value $u(t) \in X_{0}$ at time $t$ of the solution $u$ to problem (1) with data $\left(u_{0}, \bar{u}_{0}=\bar{u}_{1}=\bar{u}\right)$. We study the behaviour of the trajectories $S_{\bar{u}}(t) u_{0}$ as $t \rightarrow+\infty$.

4.1. Compactness.

Proposition 3. Assume that (4) holds. Let $u_{0} \in X_{0}$. Then $\left(S_{\bar{u}}(t) u_{0}\right)_{t>0}$ is relatively compact in $X_{0}$.

Proof. Suppose first that $u_{0} \in X_{0}$ additionally satisfies $u_{0} \in B V(0,1)$. By (10), $\left(S_{\bar{u}}(t) u_{0}\right)_{t>0}$ is bounded in $L^{1} \cap B V(0,1)$ and therefore relatively compact in $L^{1}(0,1)$. In the general case, fix $\varepsilon>0$, choose $u_{0}^{\varepsilon} \in X_{0} \cap B V(0,1)$ and $\left\|u_{0}-u_{0}^{\varepsilon}\right\|_{L^{1}(0,1)} \leq \varepsilon$ $\left(u_{0}^{\varepsilon}:=\widetilde{u_{0}} \star \rho_{\varepsilon}\right.$ where $\widetilde{u_{0}}$ is the extension of $u_{0}$ by 0 outside $[0,1]$ and $\rho_{\varepsilon}$ a classical (nonnegative) approximation of unity will do). Then $\left\|S_{\bar{u}}(t) u_{0}^{\varepsilon}-S_{\bar{u}}(t) u_{0}\right\| \leq \varepsilon$ for every $t$ by (9). Therefore, for every $\varepsilon>0$, the set $E:=\left\{S_{\bar{u}}(t) u_{0} ; t>0\right\}$ is at a distance less than $\varepsilon$ of a set $E_{\varepsilon}\left(=\left\{S_{\bar{u}}(t) u_{0}^{\varepsilon} ; t>0\right\}\right)$ which is relatively compact in $X_{0}$. Being relatively compact, each $E_{\varepsilon}$ is totally bounded, and therefore so is $E$. Since $X_{0}$ is a Banach space, the set $E$ is relatively compact in $X_{0}$.

As a consequence of Proposition 3, each $\omega$-limit set

$$
\omega\left(u_{0}\right):=\bigcap_{t>0} \overline{\left\{S_{\bar{u}}(\tau) u_{0} ; \tau \geq t\right\}}
$$

is non-empty, for $u_{0} \in X_{0}$. Let $\omega\left(X_{0}\right)$ be the union of the $\omega$-limit sets:

$$
\omega\left(X_{0}\right):=\bigcap_{t>0} \overline{\left\{S_{\bar{u}}(\tau) X_{0} ; \tau \geq t\right\}}
$$

We now study $\omega\left(X_{0}\right)$.

\subsection{Bounds on the adherence values.}

Lemma 9. Assume that (4)-(6) hold. Let $\bar{q}=A(0, \bar{u})$, let $q=\min \left(\bar{q}, q_{\lim }\right)$ and let $\lambda$ and $\mu$ be the elementary solutions of the equation $A(x, w(x))=q$ (see Lemma 6 ). Then we have

$$
\omega\left(X_{0}\right) \subset\left\{w \in X_{0}, \lambda \leq w \text { a.e. }\right\} .
$$

If, furthermore, $Q \leq q$ (in which case $\mu$ is different from $\lambda$ ), we have

$$
\omega\left(X_{0}\right) \subset\left\{w \in X_{0}, \lambda \leq w \leq \mu \text { a.e. }\right\} \text {. }
$$

Proof. If $\bar{q}=0$, then $q=0, \lambda=0$ and (17) is obvious. Therefore, suppose that $\bar{q}>0$. We have $q>0$ and $\lambda>0$. For small $\varepsilon>0$, let $q-\varepsilon<q_{\varepsilon}<q$ and let $\lambda_{\varepsilon}$ be the first continuous branch of the equation $A(x, w(x))=q_{\varepsilon}$. Since $q_{\varepsilon}<q$, we have $\lambda_{\varepsilon}<\lambda$, and since $\lambda \leq \alpha$, we also have $\lambda_{\varepsilon}<\alpha$ on $[0,1]$. From hypothesis (5), it follows that

$$
A_{u}(x, \sigma)>0, \quad \forall(x, \sigma) \in E:=\left\{(x, \sigma) \in[0,1]^{2} ; 0 \leq \sigma \leq \lambda_{\varepsilon}(x)\right\} .
$$


By continuity of $\lambda_{\varepsilon}$, the set $E$ is closed, hence compact in $[0,1]^{2}$. By continuity of $A_{u}$ then defining $\gamma:=\min _{(x, \sigma) \in E} A_{u}(x, \sigma)>0$, it follows that

$$
\Phi^{+}\left(x, \lambda_{\varepsilon}(x), w\right)=\operatorname{sgn}_{+}\left(\lambda_{\varepsilon}(x)-w\right) \int_{w}^{\lambda_{\varepsilon}(x)} A_{u}(x, \sigma) d \sigma \geq \gamma\left(\lambda_{\varepsilon}(x)-w\right)^{+},
$$

for all $x, w \in[0,1]$. The function $\lambda_{\varepsilon}$ is a solution, hence a sub-solution of problem (1) with the boundary datum $\lambda_{\varepsilon}(0)$. From (8) we deduce, for $u_{0} \in X_{0}, \varphi$ a non-negative test function in $\mathcal{C}_{c}^{\infty}\left(\mathbb{R}^{2}\right)$,

$$
\begin{aligned}
\int_{0}^{1} \int_{0}^{\infty}\left(\lambda_{\varepsilon}-S_{\bar{u}}(t) u_{0}\right)^{+} \varphi_{t}+\Phi^{+} & \left(x, \lambda_{\varepsilon}, S_{\bar{u}}(t) u_{0}\right) \varphi_{x} d x d t+\int_{0}^{1}\left(\lambda_{\varepsilon}-u_{0}\right)^{+} \varphi(0, x) d x \\
& +2 \mathcal{L} \int_{0}^{\infty}\left(\lambda_{\varepsilon}(0)-\bar{u}\right)^{+}(\varphi(t, 0)+\varphi(t, 1)) d t \geq 0 .
\end{aligned}
$$

Since $\bar{q} \geq q \geq q_{\varepsilon}$ and $0 \leq \lambda_{\varepsilon}(0) \leq \alpha(0)$, we have $\left(\lambda_{\varepsilon}(0)-\bar{u}\right)^{+}=0$ and the last term in $(20)$ vanishes. Choosing $\varphi(x, t)=\alpha(t) e^{-x}$ where $\alpha$ is a non-negative function of $\mathcal{C}_{c}^{\infty}(0,+\infty)$, we have

$$
\int_{0}^{1} \int_{0}^{+\infty}\left(\left(\lambda_{\varepsilon}-S_{\bar{u}}(t) u_{0}\right)^{+} \alpha_{t}-\Phi^{+}\left(x, \lambda_{\varepsilon}, S_{\bar{u}}(t) u_{0}\right) \alpha\right) e^{-x} \geq 0 .
$$

By use of the estimate (19), we get

$$
\int_{0}^{+\infty}\left(\int_{0}^{1}\left(\lambda_{\varepsilon}-S_{\bar{u}}(t) u_{0}\right)^{+} e^{-x}\right)\left(\alpha_{t}-\gamma \alpha\right) \geq 0
$$

which gives the exponential decrease

$$
\int_{0}^{1}\left(\lambda_{\varepsilon}-S_{\bar{u}}(t) u_{0}\right)^{+} e^{-x} d x \leq\left(\int_{0}^{1}\left(\lambda_{\varepsilon}-u_{0}\right)^{+} e^{-x} d x\right) e^{-\gamma t}
$$

and the inclusion $\omega\left(X_{0}\right) \subset\left\{w \in X_{0}, \lambda_{\varepsilon} \leq w\right.$ a.e. $\}$. Since $\omega\left(X_{0}\right)$ is closed and since $\lambda \rightarrow \lambda_{\varepsilon}$ (uniformly on $[0,1]$ ) when $\varepsilon \rightarrow 0$, we get (17) at the limit $\varepsilon>0$. If $\bar{q}=q_{\text {lim }}=Q$, then $\mu=1$ and the inclusion (18) is obvious. If $q_{\lim } \geq \bar{q}>Q$, then $q=\bar{q}$ and the inclusion $\omega\left(X_{0}\right) \subset\left\{w \in X_{0}, w \leq \mu\right.$ a.e. $\}$ is obtained by similar methods as (17), on the basis of the estimate

$$
\Phi^{+}\left(x, w, \mu_{\varepsilon}(x)\right) \leq-\tilde{\gamma}\left(w-\mu_{\varepsilon}(x)\right)^{+},
$$

$(x, w) \in[0,1]^{2}, \tilde{\gamma}>0$, which holds as $\mu_{\varepsilon}$ is an upper branch of the solution for the equation $A(x, w(x))=q_{\varepsilon}, q-\varepsilon<q_{\varepsilon}<q$.

4.3. Convergence to stationary solutions. We first prove the following proposition.

Proposition 4. Assume that (2)-(6) hold. The set $\omega\left(X_{0}\right)$ is a subset of the set of stationary solutions to problem (1).

Proof. We use the result and the notation of Lemma 9. Set $\hat{\mu}:=1$ if $q<Q, \hat{\mu}:=\mu$ if $q \geq Q$, so that the conclusion of Lemma 9 reads $\omega\left(X_{0}\right) \subset X_{1}, X_{1}:=\left\{w \in X_{0}, \lambda \leq\right.$ $w \leq \hat{\mu}$ a.e. $\}$. Let $w \in \omega\left(X_{0}\right)$ and let $\hat{w}$ be a stationary sub-solution of problem (1) with boundary datum $\bar{u}$. By Proposition 1, the function $u \mapsto\left\|(\hat{w}-u)^{+}\right\|_{L^{1}}$ is nonincreasing along the trajectories drawn by $S_{\bar{u}}$. Therefore, by the LaSalle Principle, $t \mapsto\left\|\left(\hat{w}-S_{\bar{u}}(t) w\right)^{+}\right\|_{L^{1}}$ is constant. 
Similarly, if $\hat{w}$ is a stationary super-solution of problem (1) with boundary datum $\bar{u}$, we see that $t \mapsto\left\|\left(\hat{w}-S_{\bar{u}}(t) w\right)^{-}\right\|_{L^{1}}$ is constant.

Now, we use Lemma 8 to show that $S_{\bar{u}}(t) w=w$. If $q<Q$, for example, the functions $\hat{w}_{z}$ are stationary super-solutions and we have

$$
\left\|\left(\hat{w}_{z}-S_{\bar{u}}(t) w\right)^{-}\right\|_{L^{1}}=\left\|\left(\hat{w}_{z}-w\right)^{-}\right\|_{L^{1}}, \quad z \in[0,1], t>0 .
$$

Moreover, $w \in X_{1}$, and therefore

$$
\left\|(\hat{w}-w)^{-}\right\|_{L^{1}}=\int_{z}^{1}(1-w) d x .
$$

By Proposition 1 , the set $X_{1}$ is invariant under $S_{\bar{u}}$ so that we also have

$$
\left\|\left(\hat{w}-S_{\bar{u}}(t) w\right)^{-}\right\|_{L^{1}}=\int_{z}^{1}\left(1-S_{\bar{u}}(t) w\right) d x .
$$

We deduce

$$
\int_{z}^{1} w=\int_{z}^{1} S_{\bar{u}}(t) w
$$

for all $z \in[0,1], t>0$, i.e. $S_{\bar{u}}(t) w=w$.

Similarly, for all the possible values of $q$ and $\bar{u}$, we use the fact that Lemma 8 provides enough sub- (or super-) solutions to deduce $S_{\bar{u}}(t) w=w$ from the fact that $t \mapsto$ $\left\|\left(\hat{w}-S_{\bar{u}}(t) w\right)^{ \pm}\right\|_{L^{1}}$ is constant: we therefore conclude that any $w \in \omega\left(X_{0}\right)$ is a stationary solution to (1) with boundary datum $\bar{u}$.

Theorem 4. Assume that (2)-(6) hold. For every $u_{0} \in X_{0}$, the trajectory $S_{\bar{u}}(t) u_{0}$ converges to a stationary state. Stationary states are described in Theorem 3.

REMARK 6 . If $\bar{q}:=A(0, \bar{u})$ is different from $q_{\text {lim }}$, then there is only one stationary solution (see Theorem 3 ) and Theorem 4 gives the asymptotic behaviour of $S_{\bar{u}}(t) u_{0}$ : for large time, it converges to the unique stationary solution. In case $\bar{q}=q_{\text {lim }}$, the asymptotic behaviour of the trajectories depends on their starting point. It is however possible to determine explicitly the limit in some situations; see Proposition 5.

Proof. Let $u_{0} \in X_{0}$. By Proposition 3, there exists $w \in \omega\left(X_{0}\right)$. By Proposition 4, we know that $w$ is a stationary state. Together with the fact that $S_{\bar{u}}$ is non-expansive (equation (9)), this gives $S_{\bar{u}}(t) u_{0} \rightarrow w$ in $X_{0}$. Indeed, given $\varepsilon>0$, there exists a time $t_{\varepsilon}$ at which $\left\|S_{\bar{u}}\left(t_{\varepsilon}\right) u_{0}-w\right\|_{L_{1}}<\varepsilon$. Since $S_{\bar{u}}$ is $L^{1}$ non-expansive and $w$ a fixed point of $S_{\bar{u}}$, this remains true for every time $t \geq t_{\varepsilon}$. Therefore $S_{\bar{u}}(t) u_{0} \rightarrow w$ in $X_{0}$.

The following proposition completes the result stated in Theorem 4 in the case $\bar{q}=q_{\text {lim }}$ (see Remark 6).

Proposition 5. Assume that (2)-(6) hold. Furthermore, we suppose that

$$
\bar{q}:=A(0, \bar{u})=q_{\text {lim }} .
$$

Then, we have the following cases:

- if $u_{0} \leq \lambda$, then $S_{\bar{u}}(t) u_{0}$ converges to $\lambda$,

- if $u_{0} \geq \mu$, then $S_{\bar{u}}(t) u_{0}$ converges to $\mu$, 
- if $\lambda \leq u_{0} \leq \mu$ and $\bar{u}=\lambda(0)$, then $S_{\bar{u}}(t) u_{0}$ converges to $w_{z, 1}$ where $z$ is the unique element of $[0,1 / 2]$ such that

$$
\int_{0}^{1 / 2}\left(u_{0}-\lambda\right) d x=\int_{0}^{1 / 2}\left(w_{z, 1}-\lambda\right) d x
$$

- if $\lambda \leq u_{0} \leq \mu$ and $\bar{u}=\mu(0)$, then $S_{\bar{u}}(t) u_{0}$ converges to $w_{0, z^{\prime}}$ where $z^{\prime}$ is the unique element of $[1 / 2,1]$ such that

$$
\int_{1 / 2}^{1}\left(\mu-u_{0}\right) d x=\int_{1 / 2}^{1}\left(\mu-w_{0, z^{\prime}}\right) d x
$$

Notice that if $u_{0}$ is not ordered with respect to $\lambda$ and/or $\mu$, we cannot specify the asymptotic stationary state.

Proof. In the first two cases, the convergence follows from Lemma 9 and the fact that $\{w \leq \lambda\}$ and $\{w \geq \mu\}$ are invariant under $S_{\bar{u}}$. In the case $\lambda \leq u_{0} \leq \mu$ and $\bar{u}=\lambda(0)$, we observe that

$$
\int_{0}^{1 / 2} S_{\bar{u}}(t) u_{0}-\lambda=\text { constant. }
$$

This uniquely determines the elements of $\omega\left(u_{0}\right)$, already restricted to be functions $w_{z, 1}$ by Proposition 4 and Lemma 7 .

Similarly, in the case $\lambda \leq u_{0} \leq \mu$ and $\bar{u}=\mu(0)$, the $\omega$-limit set $\omega\left(u_{0}\right)$ reduces to a singleton by the constraint

$$
\int_{1 / 2}^{1} \mu-S_{\bar{u}}(t) u_{0}=\text { constant. }
$$

Let us prove (21) for example: by Lemma 8, the functions

$$
w_{1 / 2,1 / 2}=\lambda \mathbf{1}_{[0,1 / 2]}+\mu \mathbf{1}_{[1 / 2,1]}, \quad w_{0,1}=\lambda \mathbf{1}_{[1 / 2,1]}+\mu \mathbf{1}_{[0,1 / 2]}
$$

are, respectively, super- and sub-solution of problem (1). From Proposition 1, we deduce that the functions

$$
t \mapsto \int_{0}^{1}\left(S_{\bar{u}}(t) u_{0}-w_{1 / 2,1 / 2}\right)^{+}, \quad t \mapsto \int_{0}^{1}\left(w_{0,1}-S_{\bar{u}}(t) u_{0}\right)^{+}
$$

are non-increasing. Since $\{\lambda \leq w \leq \mu\}$ is invariant by $S_{\bar{u}}$, we have

$$
\int_{0}^{1 / 2} S_{\bar{u}}(t) u_{0}-\lambda=\int_{0}^{1}\left(S_{\bar{u}}(t) u_{0}-w_{1 / 2,1 / 2}\right)^{+}=\int_{0}^{1 / 2}(\mu-\lambda)-\int_{0}^{1}\left(w_{0,1}-S_{\bar{u}}(t) u_{0}\right)^{+} .
$$

Therefore

$$
t \mapsto \int_{0}^{1 / 2} S_{\bar{u}}(t) u_{0}-\lambda
$$

is at the same time non-increasing and non-decreasing, i.e. it is constant.

5. Numerical results. In this section, we focus on the numerical approximation of problem (1) with flux $A$ given by the formula

$$
A(x, u)=Q u+H(x) u(1-u) .
$$


5.1. Finite volume method for scalar conservation laws. We present the numerical method that has been used for the simulation of the model case. Let us consider a uniform mesh in space (with $N+1$ elements). We classically denote by $h=1 / N$ the mesh size, by $k$ the time step, and by $\mathcal{T}=\left\{K_{i}\right\}_{1 \leq i \leq N}$ the family of the $N$ control volumes. Let us define the following relationship:

$$
u_{i}^{n+1}=u_{i}^{n}-\frac{k}{h}\left(F_{i+1 / 2}\left(u_{i+1}^{n}, u_{i}^{n}\right)-F_{i-1 / 2}\left(u_{i}^{n}, u_{i-1}^{n}\right)\right), \quad 1 \leq i \leq N-1,
$$

under the boundary constraints $u_{0}^{n}=\bar{u}_{0}$ and $u_{N}^{n}=\bar{u}_{1}$, for all $n \in \mathbb{N}$, and under the CFL condition $\mathcal{L} k / h<1, \mathcal{L}$ being the Lipschitz constant of $A$ w.r.t. $u$. The numerical solution is then defined by

$$
\forall K_{i} \in \mathcal{T}, \forall n \in \mathbb{N}, u_{\mathcal{T}, k}(t, x)=u_{i}^{n}, \forall(t, x) \in\left[n \Delta t,(n+1) \Delta t\left[\times K_{i} .\right.\right.
$$

The choice of the scheme now relies on the numerical fluxes $F_{i \pm 1 / 2}$, which should satisfy properties of regularity, consistency, conservativity and monotonicity. Then, the numerical solution converges to the entropy solution of (1) [Vov02]. For the numerical simulations, the following scheme has been used:

Definition 5 (ENO scheme). Let us define $\widetilde{u}(x) \in(0,1]$ as the maximum of $u \mapsto$ $A(x, u)$ on the interval $[0,1]$ :

$$
\widetilde{u}(x)=\min \left(1, \frac{1}{2}\left(1+\frac{Q}{H(x)}\right)\right)
$$

and let us define the partial fluxes:

$$
\begin{aligned}
& A^{-}(x, u)= \begin{cases}A(x, u)-A(x, \widetilde{u}(x)), & \text { if } u \geq \widetilde{u}(x), \\
0, & \text { otherwise }\end{cases} \\
& A^{+}(x, u)= \begin{cases}A(x, \widetilde{u}(x)), & \text { if } u \geq \widetilde{u}(x), \\
A(x, u), & \text { otherwise }\end{cases}
\end{aligned}
$$

The ENO flux is defined by the following formula:

$$
F_{i+1 / 2}(u, v)=A^{-}\left(x_{i+1}, u\right)+A^{+}\left(x_{i}, v\right) .
$$

5.2. Numerical tests. In the entire section, simulations are performed with the ENO scheme. We also apply the boundary condition $\bar{u}_{0}=\bar{u}_{1}=\bar{u}=0.35$ and we use a normalized gap which can be expressed as

$$
H(x)=\left(H_{\max }-H_{\min }\right)(2 x-1)^{2}+H_{\min } .
$$

As was pointed out in Theorem 3, different cases can be obtained according to the chosen values of $Q, H_{\min }$ and $H_{\max }$ :

$$
\begin{array}{llll}
\text { - } & \text { Case I } & : & \bar{q}<q_{\lim }, \\
\text { - } & \text { Case II } & : & Q<\bar{q}=q_{\lim }(\mathrm{II}(\mathrm{a})) \text { or } Q=\bar{q}=q_{\lim }(\mathrm{II}(\mathrm{b})) \text {, } \\
\text { - } & \text { Case III } & : & \bar{q}>q_{\mathrm{lim}} .
\end{array}
$$

In TABLE 1, we present some data corresponding to each case, in order to illustrate the behaviour of the weak entropy solution.

In order to illustrate the structural differences of the flux, Figs. 3-4 provide the graphs of $u \mapsto A(x, u)$, at different fixed $x$, for a given set of data $\left(H_{\min }, H_{\max }, Q\right)$. Actually, Fig. 3 (resp. Fig. 4) corresponds to Case I (resp. Case III). 


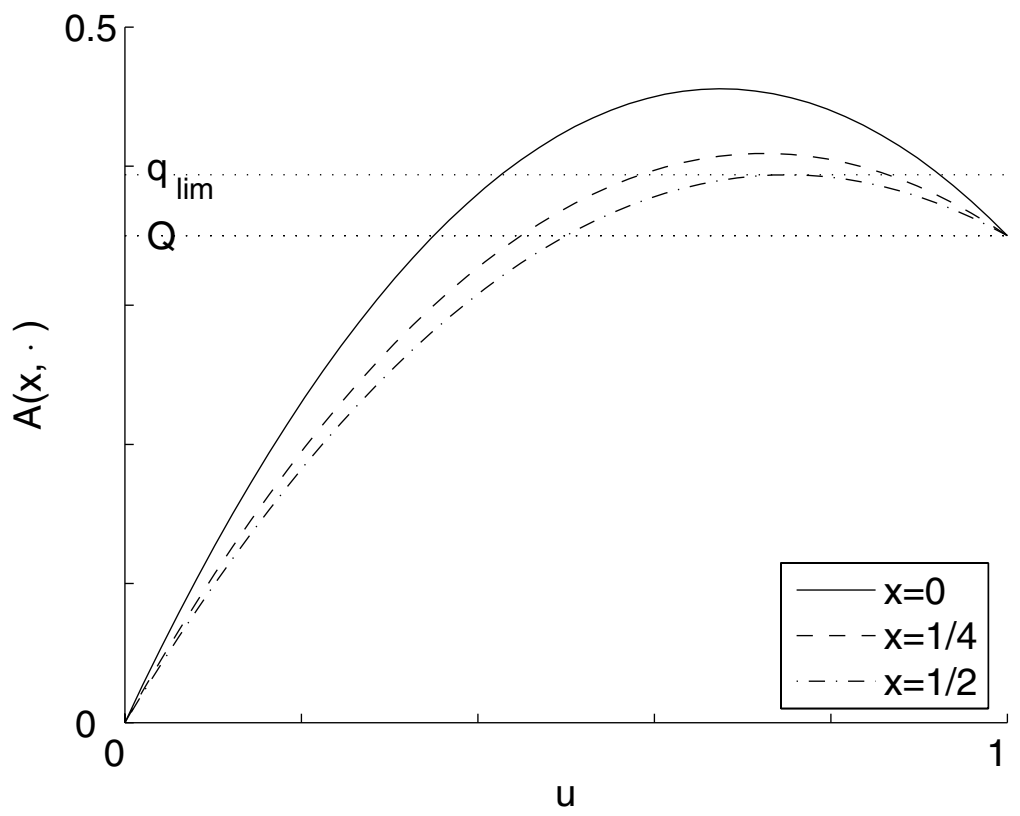

FIG. 3. Graph of $u \mapsto A(x, u)$ in case $Q<q_{\text {lim }}$.

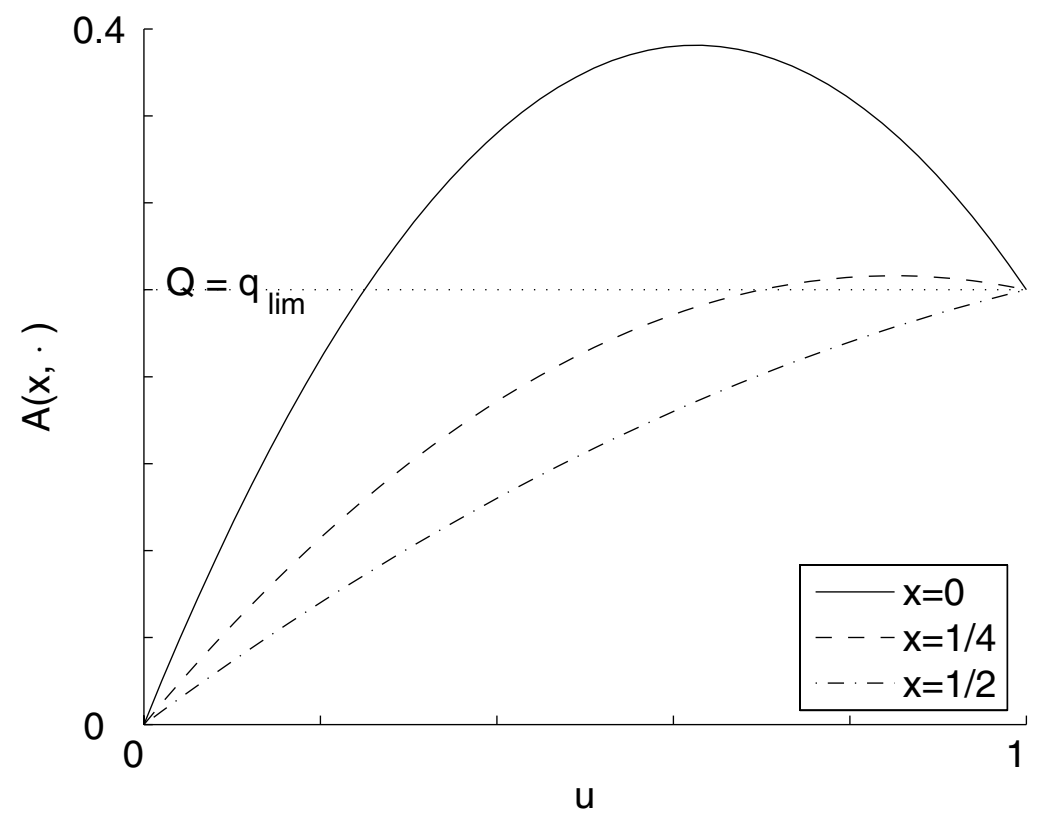

FIG. 4. Graph of $u \mapsto A(x, u)$ in case $Q=q_{\text {lim }}$. 
TABle 1. Numerical data.

\begin{tabular}{|c||c|c|c|c|}
\hline & I & II(a) & II(b) & III \\
\hline \hline$Q$ & 0.3500 & 0.2935 & 0.3500 & 0.2500 \\
\hline$H_{\min }$ & 0.7000 & 0.5871 & 0.1750 & 0.1250 \\
\hline$H_{\max }$ & 1.0000 & 1.0000 & 1.0000 & 1.0000 \\
\hline \hline $\bar{q}$ & 0.3500 & 0.3302 & 0.3500 & 0.3150 \\
\hline$q_{\lim }$ & 0.3937 & 0.3302 & 0.3500 & 0.2500 \\
\hline
\end{tabular}

Now, let us discuss the numerical results:

- In Case I, we observe that the weak entropy solution converges to the stationary one, as described in Theorem 3. Moreover, the stationary solution is continuous (therefore, numerical illustrations are omitted).

- In Case II(a), the stationary solution depends on the initial one. Computations illustrate the theoretical results described in Theorem 3. In particular, according to the choice of the initial solution, a discontinuous stationary solution may appear:

○ Figs. 5-6: The initial condition is defined by $u_{0}=u_{0}^{(1)} \equiv \bar{u}$. Then, we observe that $S(t)\left(u_{0}^{(1)}, \bar{u}\right)$ obviously converges to $\lambda$, since $u_{0}^{(1)} \leq \lambda$ and $\lambda(0)=\bar{u}$.

○ Figs. 7-8: The initial condition is defined by $u_{0}=u_{0}^{(2)}$ with

$$
u_{0}^{(2)}(x)=\left(1-\frac{1}{10} \sin (2 \pi x)\right) \lambda\left(\frac{1}{2}\right), \quad \text { i.e. } \quad \lambda \leq u_{0} \leq \mu .
$$

As $\lambda(0)=\bar{u}, S(t)\left(u_{0}^{(2)}, \bar{u}\right)$ converges to some $w_{z, 1}=\lambda \mathbf{1}_{[0, z) \cup[1 / 2,1]}+\mu \mathbf{1}_{(z, 1 / 2]}$ with $0 \leq z \leq 1 / 2$. The position of the stationary shock satisfies

$$
\int_{0}^{1 / 2} w_{z, 1}=\int_{0}^{1 / 2} u_{0}^{(2)}
$$

i.e. we obtain the conservativity of the mass on [0,1/2] (see Proposition 5).

- In Case II(b), Figs. 9-10 give a simulation in the critical case. The initial condition is defined by $u_{0}=u_{0}^{(3)}$ with

$$
u_{0}^{(3)}(x)= \begin{cases}\lambda(x)+3 x(\mu(x)-\lambda(x))(1+\sin (6 \pi x)), & \text { if } 0 \leq x<\frac{1}{3}, \\ \mu(x), & \text { if } \frac{1}{3} \leq x<\frac{9}{10}, \\ \lambda(x), & \text { if } \frac{9}{10} \leq x \leq 1 .\end{cases}
$$

On $[0,1 / 2]$, the initial solution lies between $\lambda$ and $\mu$. As mentioned, the mass is conserved on $[0,1 / 2]$ :

$$
\int_{0}^{1 / 2} w_{z, 1}=\int_{0}^{1 / 2} u_{0}^{(4)}
$$


On $[1 / 2,1]$, the initial solution is a (non-entropy) stationary solution, the discontinuity being decreasing (and, therefore, non-admissible). As a consequence, this profile is not preserved as $t$ tends to $+\infty$.

- In Case III, Figs. 11-12 evidence the behaviour of the entropy solution, which converges to $w_{0,1}$. Indeed, for $\lambda(0) \leq \bar{u} \leq \mu(0)$, starting from the initial solution $u_{0} \equiv \bar{u}$, we may observe on $(0,1 / 2)$ the formation of an unstationary shock which goes out from the domain: the discontinuity has been stabilized on the boundary $x=0$.
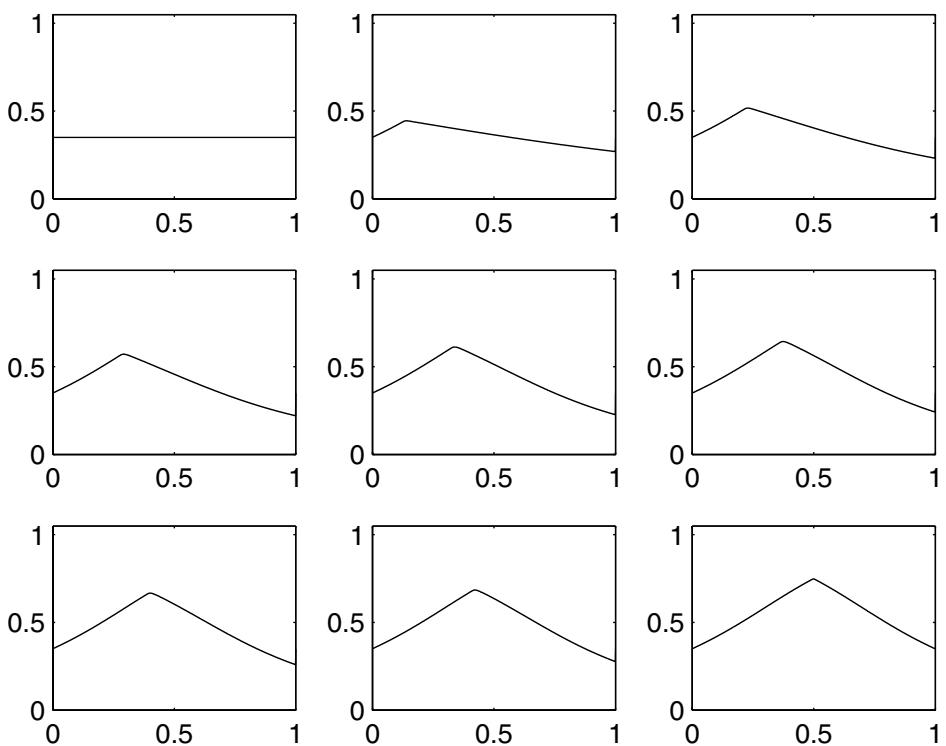

FIG. 5. Case II(a). Entropy solution at different time steps (initial condition $\left.u_{0}^{(1)}\right)$.

Finally, in TABLE 2, we illustrate Proposition 5. Notice that TABLE 2 includes information on an additional numerical simulation (for which figures have been omitted) falling into the scope of Case II(a), with the initial solution $u_{0}=u_{0}^{(4)}$ defined by

$$
u_{0}^{(4)}(x)= \begin{cases}\frac{1}{2}(\lambda(x)+\mu(x)), & \text { if } 0 \leq x<\frac{1}{2} \\ 1, & \text { if } \frac{1}{2} \leq x \leq 1\end{cases}
$$

More precisely, denoting

$$
m_{i}=\int_{0}^{1 / 2} u_{0}-\lambda, \quad m_{f}=\int_{0}^{1 / 2} w_{z, 1}-\lambda,
$$

we obtain the following results:

Among the chosen set of numerical simulations, we observe the following. 


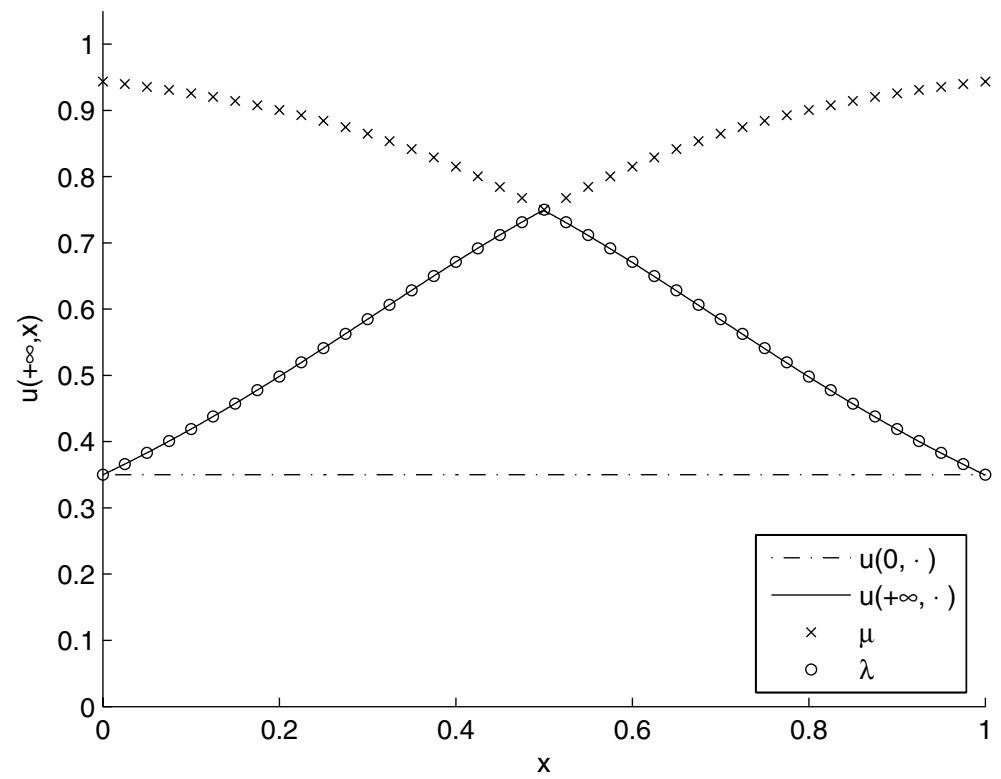

FIG. 6. Case II(a). Stationary entropy solution (initial condition $u_{0}^{(1)}$ ).
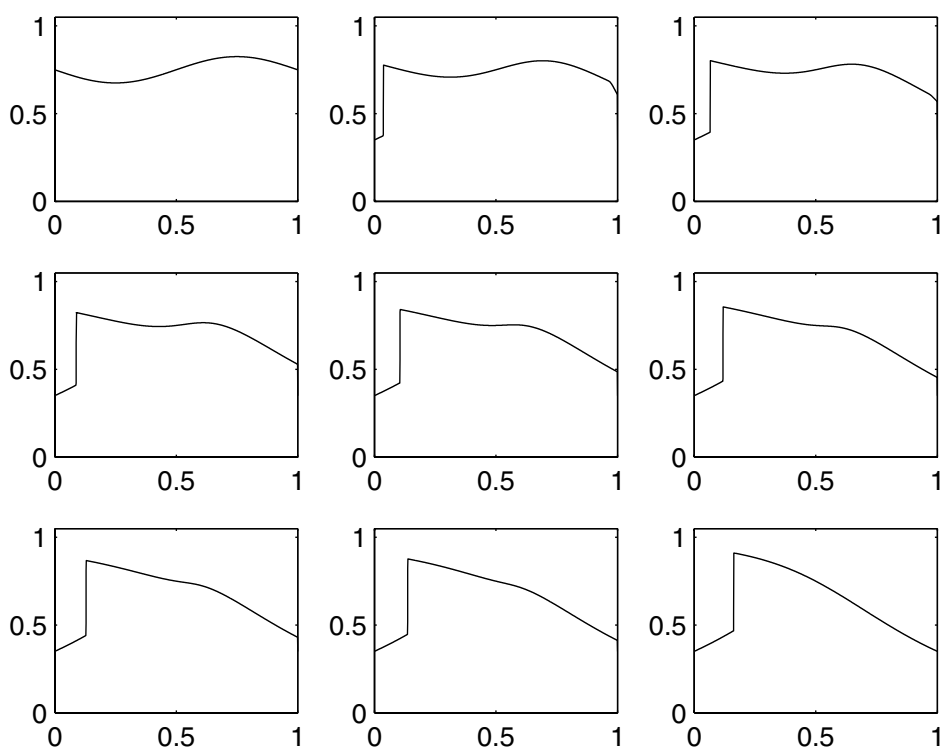

FIG. 7. Case II(a). Entropy solution at different time steps (initial condition $\left.u_{0}^{(2)}\right)$. 


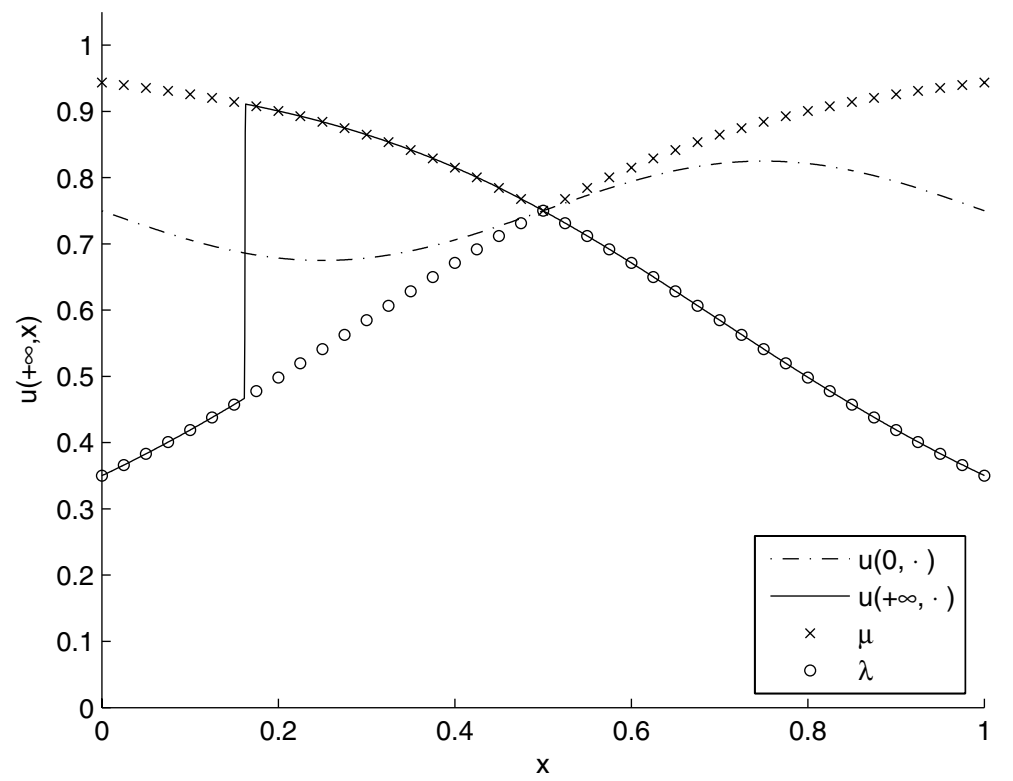

FIG. 8. Case II(a). Stationary entropy solution (initial condition $u_{0}^{(3)}$ ).
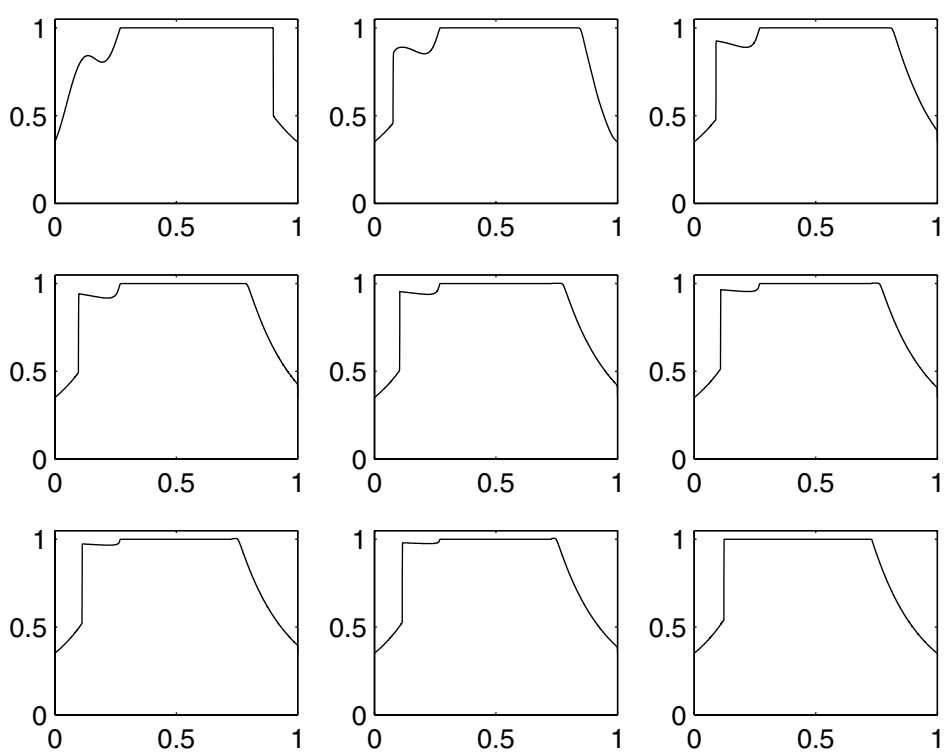

FIG. 9. Case II(b). Entropy solution at different time steps (initial condition $\left.u_{0}^{(3)}\right)$. 


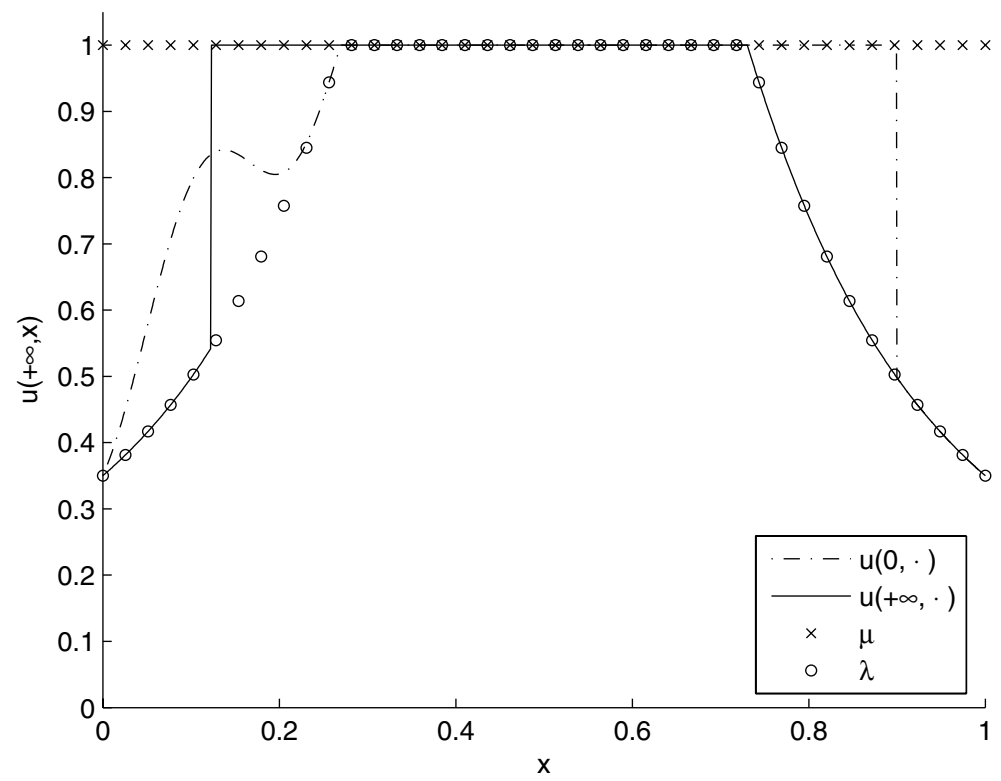

FIG. 10. Case II(b). Stationary entropy solution (initial condition $u_{0}^{(4)}$ ).
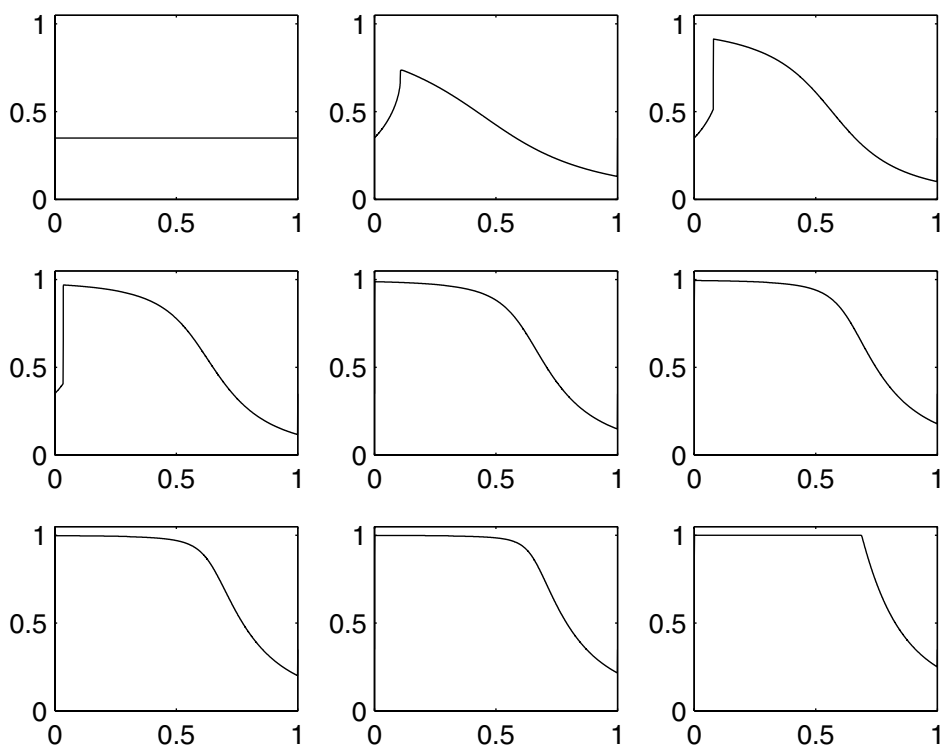

FIG. 11. Case III. Entropy solution at different time steps (initial condition $\left.u_{0}^{(1)}\right)$. 


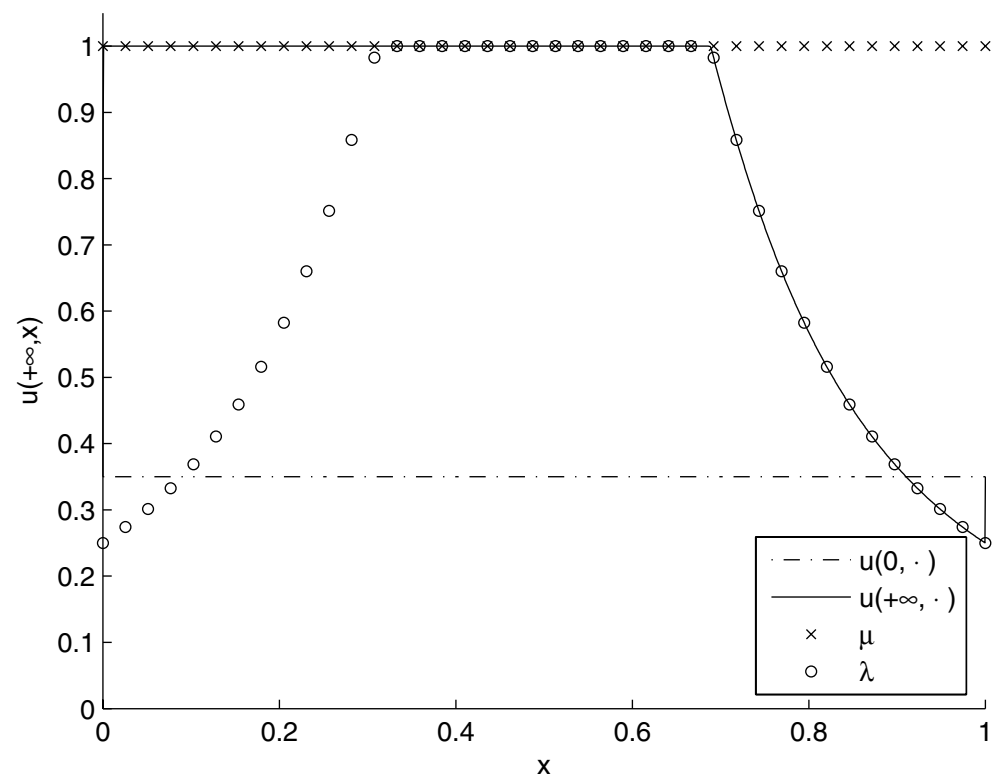

FIG. 12. Case III. Stationary entropy solution (initial condition $u_{0}^{(1)}$ ).

TABLE 2. Position of the stationary shock in Case II.

\begin{tabular}{|c||c|c|c|c|}
\hline & $\mathrm{II}(\mathrm{a}): u_{0}^{(1)}$ & $\mathrm{II}(\mathrm{a}): u_{0}^{(2)}$ & $\mathrm{II}(\mathrm{b}): u_{0}^{(3)}$ & $\mathrm{II}(\mathrm{a}): u_{0}^{(4)}$ \\
\hline \hline$m_{i}$ & $-9.079110^{-2}$ & $8.514710^{-2}$ & $3.491810^{-2}$ & $8.809910^{-2}$ \\
\hline$m_{f}$ & $6.310510^{-3}$ & $8.506010^{-2}$ & $3.478510^{-2}$ & $1.520510^{-1}$ \\
\hline$\left(m_{f}-m_{i}\right) / m_{i}$ & -1.0695 & $-1.025910^{-3}$ & $-3.831810^{-3}$ & $7.259410^{-1}$ \\
\hline
\end{tabular}

- In the Cases II(a) with $u_{0}=u_{0}^{(2)}$ and II(b) with $u_{0}=u_{0}^{(3)}$, the integral equality $m_{i}=m_{f}$ is numerically satisfied with a relative error which is less than $0.5 \%$. Indeed, the initial solution satisfies the assumption $\lambda \leq u_{0} \leq \mu$,

- In the Cases II(a) with $u_{0}=u_{0}^{(1)}$ or $u_{0}=u_{0}^{(2)}$, the integral equality $m_{i}=m_{f}$ does not hold, as the condition $\lambda \leq u_{0} \leq \mu$ is not satisfied.

\section{REFERENCES}

[BLN79] C. Bardos, A. Y. LeRoux, and J.-C. Nédélec, First order quasilinear equations with boundary conditions, Comm. Partial Differential Equations 4 (1979), no. 9, 1017-1034. MR542510 (81b:35052)

[BMV05] G. Bayada, S. Martin, and C. Vazquez, About a generalized Buckley-Leverett equation and lubrication multifluid flow, European J. Appl. Math. 17, no. 5, 491-524 (2006). MR2296026

[BC82] G. Bayada, and M. Chambat, Analysis of a free boundary problem in partial lubrication, Quart. Appl. Math. 40 (1982/83), no. 4, 369-375. MR693872 (84f:76031)

[CR00] G.-Q. Chen and M. Rascle, Initial layers and uniqueness of weak entropy solutions to hyperbolic conservation laws, Arch. Ration. Mech. Anal. 153 (2000), no. 3, 205-220. MR1771520 (2001f:35250) 
[Car99] J. Carrillo, Entropy solutions for nonlinear degenerate problems, Arch. Ration. Mech. Anal. 147 (1999), no. 4, 269-361. MR1709116 (2000m:35132)

[Daf85] C. M. Dafermos, Regularity and large time behaviour of solutions of a conservation law without convexity, Proc. Roy. Soc. Edinburgh Sect. A 99 (1985), no. 3-4, 201-239. MR785530 (86j:35107)

[DiP75] R.J. DiPerna, Decay and asymptotic behavior of solutions to nonlinear hyperbolic systems of conservation laws, Indiana Univ. Math. J. 24 (1974/75), no. 11, 1047-1071. MR0410110 (53:13860)

[Do63] G.I. Dowson, Cavitation of a viscous fluid in narrow passages, J. Fluid Mech. Math. 16 (1963), 595-619.

[EA75] H.G. Elrod and M.L Adams, A computer program for cavitation, Cavitation and related phenomena in lubrication - Proceedings - Mech. Eng. Publ. Ltd (1975), 37-42.

[EGH00] R. Eymard, T. Gallouët, and R. Herbin, Finite volume methods, Handbook of numerical analysis, Vol. VII, North-Holland, Amsterdam, 2000, pp. 713-1020. MR1804748 (2002e:65138)

[EGV03] R. Eymard, T. Gallouët, and J. Vovelle, Limit boundary conditions for finite volume approximations of some physical problems, J. Comput. Appl. Math. 161 (2003), no. 2, 349-369. MR2017019 (2005k:76083)

[FS01] H. Freistühler and D. Serre, The $L^{1}$-stability of boundary layers for scalar viscous conservation laws, J. Dynam. Differential Equations 13 (2001), no. 4, 745-755. MR1860284 (2002j:35206)

[IO60] A. M. Il'in and O. A. Oleinik, Asymptotic behavior of solutions of the Cauchy problem for some quasi-linear equations for large values of the time, Mat. Sb. (N.S.) 51 (93) (1960), 191-216. MR0120469 (22:11222)

[Kaa99] E. F. Kaasschieter, Solving the Buckley-Leverett equation with gravity in a heterogeneous porous medium, Comput. Geosci. 3 (1999), no. 1, 23-48. MR1696184 (2000b:76103)

[Kim03] Y.J. Kim, Asymptotic behavior of solutions to scalar conservation laws and optimal convergence orders to $N$-waves, J. Differential Equations 192 (2003), no. 1, 202-224. MR1987091 (2004e:35147)

[KM85] S. Kawashima and A. Matsumura, Asymptotic stability of traveling wave solutions of systems for one-dimensional gas motion, Comm. Math. Phys. 101 (1985), no. 1, 97-127. MR814544 (87h:35035)

[Lax57] P. D. Lax, Hyperbolic systems of conservation laws. II, Comm. Pure Appl. Math. 10 (1957), 537-566. MR0093653 (20:176)

[LN97] T.-P. Liu and K. Nishihara, Asymptotic behavior for scalar viscous conservation laws with boundary effect, J. Differential Equations 133 (1997), no. 2, 296-320. MR1427855 (97j:35092)

[LP84] T.-P. Liu and M. Pierre, Source-solutions and asymptotic behavior in conservation laws, J. Differential Equations 51 (1984), no. 3, 419-441. MR735207 (85i:35094)

[Mar05] S. Martin, First order quasilinear equations with boundary conditions in the $L^{\infty}$ framework, J. Diff. Equations 236, no. 2, 375-406 (2007).

[MN94] A. Matsumura and K. Nishihara, Asymptotic stability of traveling waves for scalar viscous conservation laws with non-convex nonlinearity, Comm. Math. Phys. 165 (1994), no. 1, 83-96. MR1298944 (95g:35120)

[MNRR96] J. Málek, J. Necas, M. Rokyta, and M. Ruzicka, Weak and measure-valued solutions to evolutionary PDEs, Chapman \& Hall, London, 1996.

[MT99] C. Mascia and A. Terracina, Large-time behavior for conservation laws with source in a bounded domain, J. Differential Equations 159 (1999), no. 2, 485-514. MR1730729 (2001b:35206)

[NPD97] A. Nouri, F. Poupaud, and Y. Demay, An existence theorem for the multi-fluid Stokes problem, Quart. Appl. Math. 55 (1997), no. 3, 421-435. MR1466141 (98h:35190)

[Ott93] F. Otto, Initial-boundary value problem for a scalar conservation law, $\mathrm{Ph}$. D. Thesis (1993).

[Ott96] F. Otto, Initial-boundary value problem for a scalar conservation law, C. R. Acad. Sci. Paris Sér. I Math. 322 (1996), no. 8, 729-734. MR1387428 (97c:35132)

[Pao03] L. Paoli, Asymptotic behavior of a two fluid flow in a thin domain: from Stokes equations to Buckley-Leverett equation and Reynolds law, Asymptot. Anal. 34 (2003), no. 2, 93-120. MR1992280 (2004j:35234) 
[Ser96] D. Serre, Systèmes de lois de conservation. II, Diderot Editeur, Paris, 1996. MR1459989 (99e:35144)

[Ser04] D. Serre, $L^{1}$-stability of nonlinear waves in scalar conservation laws, Evolutionary equations. Vol. I, Handb. Differ. Eq., North-Holland, Amsterdam, 2004, pp. 473-553. MR2103702 (2006f:35177)

[Vas01] A. Vasseur, Strong traces for solutions of multidimensional scalar conservation laws, Arch. Ration. Mech. Anal. 160 (2001), no. 3, 181-193. MR1869441 (2002h:35186)

[Vov02] J. Vovelle, Convergence of finite volume monotone schemes for scalar conservation laws on bounded domains, Num. Math. 90 (2002), no. 3, 563-596. MR1884231 (2002k:65158) 